\title{
Projective studies of spin nematics in a quantum frustrated ferromagnet
}

\author{
Ryuichi Shindou, ${ }^{1,2}$ Seiji Yunoki, ${ }^{3,4,5}$ and Tsutomu Momoi ${ }^{1}$ \\ ${ }^{1}$ Condensed Matter Theory Laboratory, RIKEN, 2-1 Hirosawa, Wako, Saitama 351-0198, Japan \\ ${ }^{2}$ Physics Department, Tokyo Institute of Technology, \\ 2-12-1 Ookayama, Meguro-ku, Tokyo 152-8551, Japan \\ ${ }^{3}$ Computational Condensed Matter Laboratory, RIKEN ASI, 2-1 Hirosawa, Wako, Saitama 351-0198, Japan \\ ${ }^{4}$ Computational Materials Science Research Team, RIKEN AICS, Kobe, Hyogo 650-0047, Japan \\ ${ }^{5}$ CREST, Japan Science and Technology Agency (JST), Kawaguchi, Saitama 332-0012, Japan
}

(Dated: November 7, 2018)

\begin{abstract}
We study the ground state properties of the spin- $\frac{1}{2}$ frustrated ferromagnetic $J_{1}-J_{2}$ Heisenberg model on the square lattice, employing projected BCS wavefunctions with spin-triplet pairings of the spinon fields as trial wavefunctions. Based on the variational Monte Carlo analysis, we argue that, in the competing coupling regime, a certain type of the projected BCS wavefunction, dubbed the projected $Z_{2}$ planar state, achieves the best optimal energy among the other competing states such as the ferromagnetic state and collinear antiferromagnetic state. Like in quantum spin liquids, the projected $Z_{2}$ planar state preserves the translational symmetry of the square lattice. However, it is also accompanied by a $d$-wave ordering of the quadrupole moments, breaking the spin rotational symmetry. The state thus describes a quantum spin analogue of the nematic liquid crystals. The calculated static correlation functions also reveal that the projected $Z_{2}$ planar state has a strong collinear antiferromagnetic fluctuation.
\end{abstract}

PACS numbers:

\section{INTRODUCTION}

Deciphering unidentified states of quantum zero-point motion - quantum vacuum - is one of the central research field in condensed matter physics $\stackrel{1}{\underline{1}}$ In the realm of quantum magnetism, investigations of spin-rotational symmetric quantum spin liquid (QSL) - a quantum magnet which remains totally disordered all the way down to the zero temperature - belong to this research category. Indeed, after seminal proposal of spin-singlet resonating valence bond (RVB) wavefunctions by Anderson and his co-workers, $\stackrel{2}{=}$ tremendous research efforts are devoted to establishing a true realization of QSL or RVBtype ground state in spatial dimension greater than one $\underline{3}$ Among others, 'frustrated' Mott insulating magnets are regarded as promising candidate materials for this investigation, $\stackrel{4}{=}$ where competing magnetic interactions between localized spins often make it hard for a system to fall into a simple classical spin ordering.

This work reports a variational study of spin- $\frac{1}{2}$ quantum frustrated ferromagnetic model. One motivation of this research is a couple of experimental works on twodimensional films of solid ${ }^{3} \mathrm{He}, \stackrel{5,6}{\stackrel{5}{5}}$ where the interactions between $S=\frac{1}{2}$ nuclear spins of ${ }^{3} \mathrm{He}$ atoms are highly frustrated but predominantly ferromagnetic $\underline{\underline{z}}$ The specific heat measurement $\mathrm{t}^{-}$and magnetic susceptibility $\underline{\underline{6}}$ at the ultra-low temperature regime conclude that the ground state of this frustrated quantum spin system is a QSL-like state with either a gapless spin excitation or an extremely small spin gap. These experiments suggest a possibility of an exotic quantum phase in quantum frustrated ferromagnets.

The other incentive of this work stems from recent theoretical studies of frustrated magnets with ferromag- netic nearest-neighbor interactions on the square lattice and triangular lattice that report possible realization of spin nematic phases $\stackrel{\underline{-8}-13}{=}$ Among frustrated ferromagnets, the spin- $\frac{1}{2}$ square lattice $J_{1}-J_{2}$ model with ferromagnetic $J_{1}$ is a prototype minimal model and attracting interest recently $\underline{\underline{9}}-11,14-20$ The model Hamiltonian

$$
H=J_{1} \sum_{\langle\boldsymbol{j}, \boldsymbol{m}\rangle} \boldsymbol{S}_{\boldsymbol{j}} \cdot \boldsymbol{S}_{\boldsymbol{m}}+J_{2} \sum_{\langle\langle\boldsymbol{j}, \boldsymbol{m}\rangle\rangle} \boldsymbol{S}_{\boldsymbol{j}} \cdot \boldsymbol{S}_{\boldsymbol{m}}
$$

consists of nearest-neighbor ferromagnetic exchange $J_{1}(<0)$ and competing next-nearest-neighbor antiferromagnetic exchange $J_{2}(>0)$. When the antiferromagnetic coupling is much stronger than that of the ferromagnetic coupling $\left(\left|J_{1}\right| \ll 2 J_{2}\right)$, the ground state exhibits a collinear antiferromagnetic order, $\left\langle\boldsymbol{S}_{\boldsymbol{j}}\right\rangle=(-1)^{j_{x}} \boldsymbol{m}$ or $(-1)^{j_{y}} \boldsymbol{m}$ with $\boldsymbol{j}=\left(j_{x}, j_{y}\right) \underline{\underline{21}}$ While the ground state in the opposite limit $\left(\left|J_{1}\right| \gg 2 J_{2}\right)$ is the fully polarized ferromagnetic state. The preceding exact diagonalization studies ${ }^{\underline{9}} \underline{-11}$ suggest the existence of the spin nematic phase between these two magnetic ordered phases.

Motivated by these studies, the present authors recently formulated a fermionic mean-field theory for quantum frustrated ferromagnets, $\frac{18}{2}$ where they proposed to describe the quantum spin nematic phase as a spin-triplet variant of spin-rotational symmetric quantum spin liquids. The state suggested by this mean-field theory is a 'mixed' resonating valence bond (RVB) wavefunction, where all the half-spins in any lattice points belong to either the singlet valence bonds on antiferromagnetic links or the spin-triplet valence bonds introduced on ferromagnetic links. The wavefunction is given by a superposition of different partitioning of spins into either singlet or triplet bonds, such that the wavefunction, on the whole, has no preference for any specific valence bond configu- 
ration

Unlike Néel-ordered states or dimer states, this mixed RVB state preserves the lattice-translational symmetry of the square lattice, indicating its 'quantum spin liquid' like character. In contrast to the spin-singlet RVB state, however, the spin-1 moment in the triplet valence bond breaks spin-rotational symmetry, quantummechanically rotating within a specific plane. Thus, unlike spin-rotational symmetric quantum spin liquids, this mixed RVB state is accompanied by the breakdown of global spin-rotational symmetry. In fact, this spontaneous symmetry breaking manifests itself as the ordering of the quadrupole moment,,$\frac{8,9,18,22}{2}$

$$
K_{\boldsymbol{j}, \boldsymbol{m}}^{\mu \nu} \equiv \frac{1}{2}\left(S_{\boldsymbol{j}, \mu} S_{\boldsymbol{m}, \nu}+S_{\boldsymbol{j}, \nu} S_{\boldsymbol{m}, \mu}\right)-\frac{\delta_{\mu \nu}}{3}\left\langle\boldsymbol{S}_{\boldsymbol{j}} \cdot \boldsymbol{S}_{\boldsymbol{m}}\right\rangle,
$$

instead of the ordering of the spin dipole moment, i.e., $\left\langle\boldsymbol{S}_{\boldsymbol{j}}\right\rangle=\mathbf{0}$. In Eq. (2), $\boldsymbol{j}$ and $\boldsymbol{m}$ denote two adjacent lattice sites connected by a ferromagnetic link, and $\mu$ and $\nu$ are the spin indices. Having both quantum spin liquid character and also symmetry breaking phase character, this mixed RVB state is regarded as the quantum spin analogue of 'liquid-crystal' like state of matter $\frac{8,9,18,22,23}{2}$

To investigate the nature of this unconventional quantum spin state, we study, in this paper, the spin- $\frac{1}{2}$ square lattice $J_{1}-J_{2}$ Heisenberg model, using the variational Monte Carlo method. Our method is based on the fermionic representation of spin- $\frac{1}{2}$ operators $S_{j, \mu}=$ $\frac{1}{2} f_{j, \alpha}^{\dagger}\left[\sigma_{\mu}\right]_{\alpha \beta} f_{\boldsymbol{j}, \beta} \quad(\mu=1,2,3) \stackrel{1,24,25}{\stackrel{2}{2}}$ where $f_{\boldsymbol{j}, \alpha}^{\dagger}$ denotes the fermion creation operator at the site $\boldsymbol{j}$ with spin $\alpha$ and $\sigma_{\mu}$ the Pauli matrices. The representation becomes exact, when one and only one fermion is located at every site, i.e., $f_{j, \alpha}^{\dagger} f_{\boldsymbol{j}, \alpha}=1$. At the mean-field level, this local constraint on fermions' number is replaced by their coupling with the chemical potential, so that the only the global constraint is taken into account.

In this fermionic representation, the exchange interaction between two localized spins is written as a four-point interaction, which the mean-field theory decomposes into the pairing fields between two adjacent fermions. The antiferromagnetic exchange interaction is decoupled in terms of the spin-singlet pairing fields as $\underline{1,24} \underline{-26}$

$$
\begin{aligned}
& \boldsymbol{S}_{\boldsymbol{i}} \cdot \boldsymbol{S}_{\boldsymbol{j}} \simeq \frac{1}{4}\left(\left|\chi_{\boldsymbol{i} \boldsymbol{j}}\right|^{2}+\left|\eta_{\boldsymbol{i j}}\right|^{2}\right)+\mathrm{const} \\
& \quad+\frac{1}{4}\left(-\chi_{\boldsymbol{j} \boldsymbol{i}} f_{\boldsymbol{i}, \alpha}^{\dagger} f_{\boldsymbol{j}, \alpha}-\eta_{\boldsymbol{j} \boldsymbol{i}} f_{\boldsymbol{i}, \alpha}^{\dagger}\left[i \sigma_{2}\right]_{\alpha \beta} f_{\boldsymbol{j}, \beta}^{\dagger}+\text { h.c. }\right),(3)
\end{aligned}
$$

where $\chi_{i j}$ denotes the particle-hole (excitonic) pairing field,

$$
\chi_{\boldsymbol{i j}}=\left\langle f_{\boldsymbol{i}, \alpha}^{\dagger} f_{\boldsymbol{j}, \alpha}\right\rangle,
$$

and $\eta_{i j}$ is the particle-particle (Cooper) pairing field,

$$
\eta_{\boldsymbol{i j}}=\left\langle f_{\boldsymbol{i}, \alpha}\left[-i \sigma_{2}\right]_{\alpha \beta} f_{\boldsymbol{j}, \beta}\right\rangle .
$$

On the other hand, the competing ferromagnetic exchange interaction should be decoupled into the spin- triplet channel in its own right, $\underline{\underline{18}}$

$$
\begin{aligned}
- & \boldsymbol{S}_{\boldsymbol{i}} \cdot \boldsymbol{S}_{\boldsymbol{j}} \simeq \frac{1}{4}\left(\left|\boldsymbol{E}_{\boldsymbol{i} \boldsymbol{j}}\right|^{2}+\left|\boldsymbol{D}_{\boldsymbol{i} \boldsymbol{j}}\right|^{2}\right)+\text { const. } \\
& +\frac{1}{4} \sum_{\mu=1}^{3}\left(-E_{\boldsymbol{j} \boldsymbol{i}, \mu} f_{\boldsymbol{i}, \alpha}^{\dagger}\left[\sigma_{\mu}\right]_{\alpha \beta} f_{\boldsymbol{j}, \beta}+\text { h.c. }\right) \\
& +\frac{1}{4} \sum_{\mu=1}^{3}\left(-D_{\boldsymbol{j} \boldsymbol{i}, \mu} f_{\boldsymbol{i}, \alpha}^{\dagger}\left[-i \sigma_{\mu} \sigma_{2}\right]_{\alpha \beta} f_{\boldsymbol{j}, \beta}^{\dagger}+\text { h.c. }\right),
\end{aligned}
$$

where $\boldsymbol{D}_{\boldsymbol{j} \boldsymbol{m}}=\left(D_{\boldsymbol{j} \boldsymbol{m}, 1}, D_{\boldsymbol{j} \boldsymbol{m}, 2}, D_{\boldsymbol{j} \boldsymbol{m}, 3}\right)$ denotes the so-called $d$-vector of the spin-triplet Cooper pairing field, $\stackrel{29,30}{2}$

$$
D_{\boldsymbol{i j}, \mu}=\left\langle f_{\boldsymbol{i}, \alpha}\left[i \sigma_{2} \sigma_{\mu}\right]_{\alpha \beta} f_{\boldsymbol{j}, \beta}\right\rangle,
$$

and $\boldsymbol{E}_{\boldsymbol{j m}}=\left(E_{\boldsymbol{j m}, 1}, E_{\boldsymbol{j} \boldsymbol{m}, 2}, E_{\boldsymbol{j} \boldsymbol{m}, 3}\right)$ is the excitonic counterpart of the $d$-vector,

$$
E_{\boldsymbol{i j , \mu}}=\left\langle f_{\boldsymbol{i}, \alpha}^{\dagger}\left[\sigma_{\mu}\right]_{\alpha \beta} f_{\boldsymbol{j}, \beta}\right\rangle .
$$

Physically speaking, this decoupling is because the ferromagnetic exchange interaction between two spin halves $\frac{18,27}{}$ usually prefers the formation of their spintriplet valence bond, $\left|S=1, S_{\mu}=0\right\rangle(\mu=1,2,3)$, instead of the singlet valence bond. Indeed, the $d$-vector associated with the triplet pairing specifies the direction along which the spin triplet state has the zero magnetization, i.e. $\left|S=1,\left(\boldsymbol{e}_{d} \cdot \boldsymbol{S}\right)=0\right\rangle$ with $\boldsymbol{e}_{d}=\boldsymbol{D}_{j m} /\left|\boldsymbol{D}_{j m}\right|$. One can easily see at the mean-field level that the triplet pairing fields in Eqs. (7) and (8) induce the following quadrupole moment, 18

$$
\begin{aligned}
\left\langle K_{\boldsymbol{i}, \boldsymbol{j}}^{\mu \nu}\right\rangle= & -\frac{1}{2}\left(E_{\boldsymbol{i} \boldsymbol{j}, \mu} E_{\boldsymbol{i j}, \nu}^{*}-\frac{1}{3} \delta_{\mu \nu}\left|\boldsymbol{E}_{\boldsymbol{i j}}\right|^{2}\right) \\
& -\frac{1}{2}\left(D_{\boldsymbol{i j}, \mu} D_{\boldsymbol{i j}, \nu}^{*}-\frac{1}{3} \delta_{\mu \nu}\left|\boldsymbol{D}_{\boldsymbol{i j}}\right|^{2}\right)+\text { h.c.. }
\end{aligned}
$$

The previous fermionic mean-field analysis shows that the present square-lattice model, Eq. (11), has five different spin-triplet pairing states as its saddle point solutions: 28 (i) $Z_{2}$ planar state, (ii) $Z_{2}$ polar state, (iii) $S U(2)$ chiral $p$-wave state and (iv) 'flat-band' state, where the first state is accompanied by a 'coplanar' or 'd-wave' configuration of the quadrupole moments while the second and third ones support 'collinear' configurations of the quadrupole moments. Among them, $Z_{2}$ planar phase and $S U(2)$ chiral $p$-wave phase appear, having the lowest energy, in the finite range of competing coupling regime between the ferromagnetic phase and $\pi$-flux phase in the mean-field phase diagram.

In this paper, we investigate the nature and energetics of the projected BCS wavefunctions constructed from these mean-field pairing states. As the local constraint of the fermion density is not strictly observed in the meanfield theory, the BCS wavefunctions generally range over the 'extended' Hilbert space, which allows double occupancy or vacancy on a single site. To obtain a proper trial many-body wavefunction for the spin model, we first 


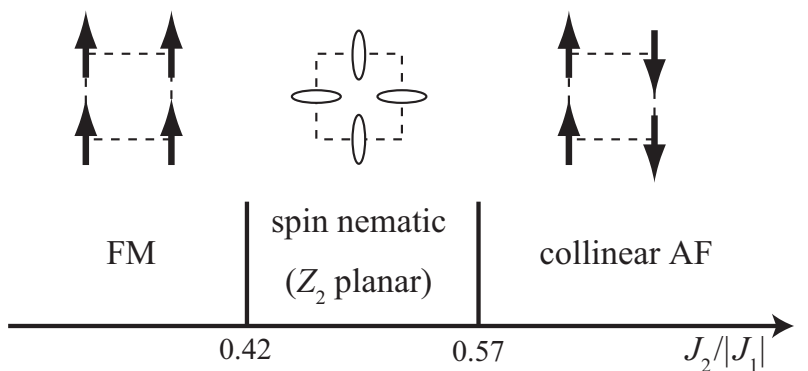

FIG. 1: Phase diagram of the spin-1/2 square lattice $J_{1}-$ $J_{2}$ model with ferromagnetic (FM) $J_{1}$ and antiferromagnetic (AF) $J_{2}$, obtained from variational Monte Carlo simulations.

project these BCS wavefunctions onto the physical spin Hilbert space, imposing the single-fermion condition on every site. By minimizing energies of these projected BCS wavefunctions in a variational way, we obtain their optimal energies. Comparing these energies with those of the ferromagnetic state and the collinear antiferromagnetic state, we argue that only a projected $Z_{2}$ planar state becomes most energetically favorable among the other competing states in a finite range of the intermediate coupling regime, $0.42\left|J_{1}\right| \lesssim J_{2} \lesssim 0.57\left|J_{1}\right|$. Our results are summarized in Fig. 1 .

Based on this observation, we further study the character of the projected $Z_{2}$ planar state. Specifically, we clarify the irreducible representations of this many-body wavefunction under the point group symmetries of the square lattice, and argue that this state is actually accompanied by a ' $d$-wave' ordering of the quadrupole moments. All irreducible representations and the $d$-wave character are totally consistent with the nature of the spin nematic phase suggested by the previous exact diagonalization study $\underline{\underline{9}}$ This agreement in combination with the energetics suggests that the projected $Z_{2}$ planar state is indeed realized in the intermediate coupling regime of the square lattice $J_{1}-J_{2}$ model. To give a direct physical characterization to this quantum spin nematic phase, we further calculate the static correlation functions in this projected BCS wavefunction in a large system size (100, 144 , and 324 sites). By that, we found that the wavefunction exhibits strong antiferromagnetic fluctuation with the wave vectors $\boldsymbol{k}=(\pi, 0)$ and $(0, \pi)$ associated with the proximate collinear antiferromagnetic phase, though the finite size scaling suggests that, the state does not possess any staggered sublattice magnetization in the thermodynamic limit.

The remaining sections of this paper are organized as follows: In Sec. II, we briefly review spin-triplet pairing states obtained by the previous fermionic mean-field analysis. We also extend these mean-field solutions into the case under finite external Zeeman field. By this extension, the projected 'flat-band' state turns out to be the fully polarized ferromagnetic state. We also find that all the $d$-vectors in the spin-triplet pairing states are lying within a plane perpendicular to the applied field. This feature guarantees the 'spin-nematic' character of the projected BCS wavefunctions constructed from these pairing states. In Sec. III, we give a general expression for the projected BCS wavefunctions having both spin-triplet and spin-singlet pairings. Based on this expression, we have optimized numerically the energies of (i) projected $Z_{2}$ planar state, (ii) projected $Z_{2}$ polar state, and (iii) projected $S U(2)$ chiral $p$-wave state. In Sec. IV, after briefly explaining the method of optimization and Monte Carlo simulations, we compare their optimized energies with other competing states such as the collinear antiferromagnetic state and the fully polarized ferromagnetic state. Sections V and VI contain discussions about the nature of the projected BCS wavefunctions. In Sec. V, we argue that all the projected BCS wavefunctions studied in this paper have a spin-nematic property, i.e., ordering of the quadrupole moments without any ordering of spins. We show in particular that the projected $Z_{2}$ planar state is accompanied by a $d$-wave spatial configuration of ordered quadrupole moments. In Sec. VI, we discuss the behavior of the static correlation functions of spins and qudrupole moments calculated in this projected $Z_{2}$ planar state. Section VII is devoted to the summary and discussion.

\section{MEAN-FIELD ANSATZ UNDER THE FIELD}

The $J_{1}-J_{2}$ frustrated ferromagnetic square lattice model has four types of spin-triplet pairing states as the saddle point solutions of the $S U(2)$ fermionic meanfield theory: (i) $Z_{2}$ planar state, (ii) $Z_{2}$ polar state, (iii) $S U(2)$ chiral $p$-wave state and (iv) 'flat-band' state, all of which possess the same translational symmetry as the square lattice. We describe in this section how these triplet pairing states are deformed under external Zeeman field. We will see that all the $d$-vectors in the states (i), (ii) and (iii) are restricted within a plane perpendicular to the applied magnetic field. Because of this arrangement, the mean-field Hamiltonian for these three states are invariant under the spin $\pi$-rotation around the field, when combined with the staggered gauge transformation $f_{\boldsymbol{j}, \alpha} \rightarrow(-1)^{j_{x}+j_{y}} f_{\boldsymbol{j}, \alpha}$. This symmetry property actually gives the spin-nematic character to the corresponding projected BCS wavefunctions (see Sec. V).

\section{A. $Z_{2}$ planar state}

In the $Z_{2}$ planar state in the absence of magnetic field, the nearest-neighbor ferromagnetic bonds support a coplanar configurations of the $d$-vector, e.g.,

$$
\begin{aligned}
D_{\boldsymbol{i j}, \mu} & = \begin{cases}D \delta_{\mu, 1} & \left(\boldsymbol{i}=\boldsymbol{j}+\boldsymbol{e}_{x}\right), \\
D \delta_{\mu, 2} & \left(\boldsymbol{i}=\boldsymbol{j}+\boldsymbol{e}_{y}\right),\end{cases} \\
E_{\boldsymbol{i j}, \mu} & =0
\end{aligned}
$$

with $\boldsymbol{e}_{x}=(1,0)$ and $\boldsymbol{e}_{y}=(0,1)$, while the nextnearest neighbor antiferromagnetic bonds support the 

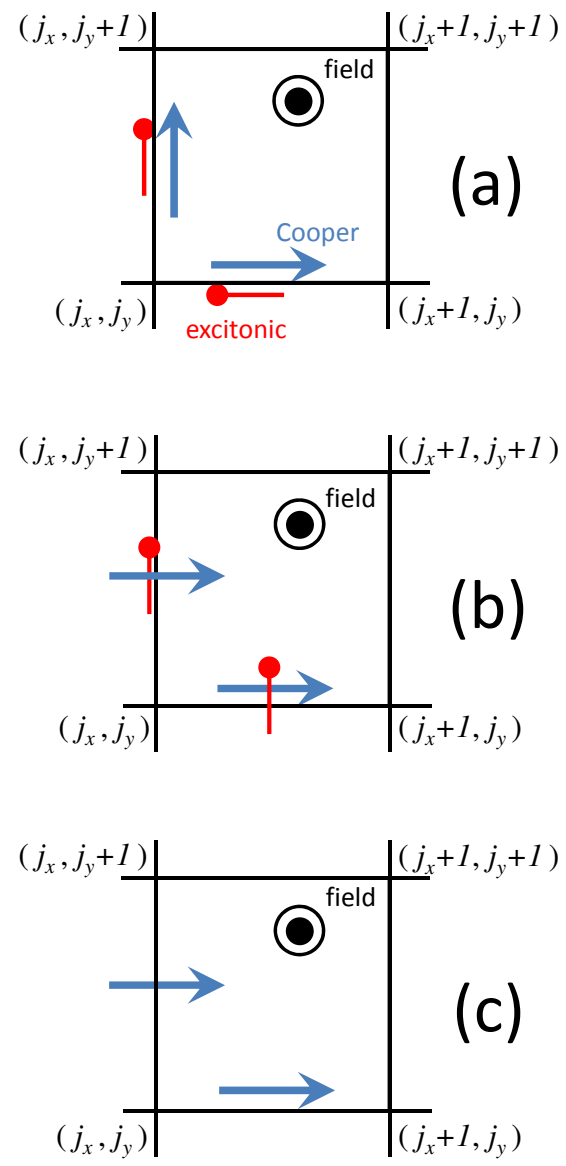

FIG. 2: (Color online) A schematic picture of the spatial configuration of $d$-vectors in (a) $Z_{2}$ planar state, (b) $Z_{2}$ polar state and (c) $S U(2)$ chiral $p$-wave state on the square lattice under the field. The $d$-vectors in the Cooper channel are drawn by (blue) arrows and those in the excitonic channel are given by (red) round head arrows, both of which are on the nearest-neighbor ferromagnetic bonds. Since all these three states preserve the translational symmetry of the square lattice, we show their configurations in the unit cell. All the $d$-vectors are lying within the plane perpendicular to the applied field. These three states are invariant under the spin $\pi$-rotation around the field, combined with staggered gauge transformation, which guarantees the spin-nematic character of the corresponding projected BCS wavefunctions (see Sec. V).

'staggered-flux' configurations of the spin-singlet pairings, 31 when $\boldsymbol{i}=\boldsymbol{j}+\boldsymbol{e}_{x} \pm \boldsymbol{e}_{y}$. The corresponding Bogoliubov de-Gennes (BdG) mean-field Hamiltonian has the form

$$
\begin{aligned}
& \mathcal{H}_{\text {planar }}=\sum_{\boldsymbol{j}}\left\{\frac{\left|J_{1}\right|}{4} D\left(-f_{\boldsymbol{j}, \alpha}^{\dagger}\left[\sigma_{3}\right]_{\alpha \beta} f_{\boldsymbol{j}+\boldsymbol{e}_{x}, \beta}^{\dagger}+i f_{\boldsymbol{j}, \alpha}^{\dagger} f_{\boldsymbol{j}+\boldsymbol{e}_{y}, \alpha}^{\dagger}\right)\right. \\
& -\frac{J_{2}}{4} \sum_{\sigma= \pm}\left(\chi f_{\boldsymbol{j}, \alpha}^{\dagger} f_{\boldsymbol{j}+\boldsymbol{e}_{x}+\sigma \boldsymbol{e}_{y}, \alpha}+i \sigma \eta f_{\boldsymbol{j}, \alpha}^{\dagger}\left[\sigma_{2}\right]_{\alpha \beta} f_{\boldsymbol{j}+\boldsymbol{e}_{x}+\sigma \boldsymbol{e}_{y}, \beta}^{\dagger}\right) \\
& \text { + h.c. }\} .
\end{aligned}
$$

This $Z_{2}$ planar state is energetically degenerate under spin $S U(2)$ rotation.

In the presence of magnetic field, spin $z$-component couples to both magnetic field and mean fields from the surrounding spins. This effect can be captured by adding the term $-h_{\mathrm{eff}} \sum_{j} S_{j, z}$ to the mean-field Hamiltonian. A direct energy optimization suggests that, under the external Zeeman field, the coplanar plane of the $d$-vectors is restricted within a plane perpendicular to the field. Moreover, the magnetic field gives rise to the excitonic triplet pairing, whose director vectors are also perpendicular to the field, replacing Eq. (9b) with

$$
E_{\boldsymbol{i j}, \mu}=\left\{\begin{array}{cl}
-i E \delta_{\mu, 1} & \left(\boldsymbol{i}=\boldsymbol{j}+\boldsymbol{e}_{x}\right), \\
i E \delta_{\mu, 2} & \left(\boldsymbol{i}=\boldsymbol{j}+\boldsymbol{e}_{y}\right) .
\end{array}\right.
$$

The mean-field Hamiltonian has the following form under the field

$$
\begin{gathered}
\mathcal{H}_{\text {planar }}^{\prime}=\mathcal{H}_{\text {planar }}+\sum_{\boldsymbol{j}}\left\{\left[i \frac { | J _ { 1 } | } { 4 } E \left(f_{\boldsymbol{j}, \alpha}^{\dagger}\left[\sigma_{1}\right]_{\alpha \beta} f_{\boldsymbol{j}+\boldsymbol{e}_{x}, \beta}\right.\right.\right. \\
\left.\left.\left.-f_{\boldsymbol{j}, \alpha}^{\dagger}\left[\sigma_{2}\right]_{\alpha \beta} f_{\boldsymbol{j}+\boldsymbol{e}_{y}, \beta}\right)+ \text { h.c. }\right]-\frac{1}{2} h_{\mathrm{eff}} f_{\boldsymbol{j}, \alpha}^{\dagger}\left[\sigma_{3}\right]_{\alpha \beta} f_{\boldsymbol{j}, \beta}\right\} .
\end{gathered}
$$

The state preserves the translational symmetry of the square lattice, so that the mean-field Hamiltonian is Fourier-transformed as

$$
\begin{aligned}
\mathcal{H}_{\text {planar }}^{\prime}=\sum_{\boldsymbol{k} ; k_{y}>0} \boldsymbol{f}_{\boldsymbol{k}}^{\dagger} & \left\{-\frac{\left|J_{1}\right|}{2} D\left(s_{x} \gamma_{3}-s_{y} \gamma_{5}\right)\right. \\
& +\frac{\left|J_{1}\right|}{2} E\left(s_{x} \gamma_{23}+s_{y} \gamma_{25}\right)-J_{2} \chi c_{x} c_{y} \gamma_{4} \\
& \left.-J_{2} \eta s_{x} s_{y} \gamma_{2}+\frac{1}{2} h_{\mathrm{eff}} \gamma_{35}\right\} \boldsymbol{f}_{\boldsymbol{k}}
\end{aligned}
$$

where $\boldsymbol{k}=\left(k_{x}, k_{y}\right), \boldsymbol{f}_{\boldsymbol{k}}^{\dagger} \equiv\left(f_{\boldsymbol{k}, \uparrow}^{\dagger}, f_{\boldsymbol{k}, \downarrow}^{\dagger}, f_{-\boldsymbol{k}, \uparrow}, f_{-\boldsymbol{k}, \downarrow}\right), f_{\boldsymbol{k}, \alpha} \equiv$ $\frac{1}{\sqrt{N}} \sum_{\boldsymbol{j}} e^{i \boldsymbol{k} \boldsymbol{j}} f_{\boldsymbol{j}, \alpha}, s_{a} \equiv \sin k_{a}$, and $c_{a} \equiv \cos k_{a}$ with $a=$ $x, y$. The $4 \times 4 \gamma$-matrices are defined as $\gamma_{1}=\sigma_{2} \otimes \sigma_{1}$, $\gamma_{2}=\sigma_{2} \otimes \sigma_{2}, \gamma_{3}=\sigma_{2} \otimes \sigma_{3}, \gamma_{4}=\sigma_{3} \otimes \sigma_{0}, \gamma_{5}=\sigma_{1} \otimes \sigma_{0}$, and $\gamma_{j m}=-i \gamma_{j} \gamma_{m}$, where the $2 \times 2$ Pauli matrices in front of the $\otimes$-mark is for the particle-hole space, while the others are for the spin space, e.g.

$$
\gamma_{1}=\left(\begin{array}{cc}
0 & -i \boldsymbol{\sigma}_{1} \\
i \boldsymbol{\sigma}_{1} & 0
\end{array}\right)
$$




\section{B. $Z_{2}$ polar state}

The $Z_{2}$ polar state at zero field takes a collinear configuration of the $d$-vectors on ferromagnetic bonds, e.g.,

$$
\begin{aligned}
D_{\boldsymbol{i j}, \mu} & =D \delta_{\mu, 1}, \\
E_{\boldsymbol{i j}, \mu} & =0
\end{aligned}
$$

for $\boldsymbol{i}=\boldsymbol{j}+\boldsymbol{e}_{a}(a=x, y)$, while it supports the same staggered-flux configuration of the singlet pairings as in Eq. (10).

In the presence of magnetic field, the external Zeeman field restricts the $d$-vectors within its transverse directions the same as in Eq. (14a) and brings about the excitonic spin-triplet pairings, replacing Eq. (14b) with

$$
E_{\boldsymbol{i j}, \mu}=i E \delta_{\mu, 2}
$$

for $\boldsymbol{i}=\boldsymbol{j}+\boldsymbol{e}_{a}(a=x, y)$. The $d$-vectors of the induced excitonic spin-triplet pairings are perpendicular to both the external field and the $d$-vectors of the Cooper channel. The mean-field Hamiltonian for the $Z_{2}$ polar state has the following form under the field,

$$
\begin{aligned}
& \mathcal{H}_{\text {polar }}=\sum_{\boldsymbol{j}}\left\{\left[\alpha_{1} f_{\boldsymbol{j}, \uparrow}^{\dagger} f_{\boldsymbol{j}, \downarrow}^{\dagger}\right.\right. \\
& -\frac{\left|J_{1}\right|}{4} \sum_{a=x, y}\left(D f_{\boldsymbol{j}, \alpha}^{\dagger}\left[\sigma_{3}\right]_{\alpha \beta} f_{\boldsymbol{j}+\boldsymbol{e}_{a}, \beta}^{\dagger}+i E f_{\boldsymbol{j}, \alpha}^{\dagger}\left[\sigma_{2}\right]_{\alpha \beta} f_{\boldsymbol{j}+\boldsymbol{e}_{a}, \beta}\right) \\
& -\frac{J_{2}}{4} \sum_{\sigma= \pm}\left(\chi f_{\boldsymbol{j}, \alpha}^{\dagger} f_{\boldsymbol{j}+\boldsymbol{e}_{x}+\sigma \boldsymbol{e}_{y}, \alpha}+i \sigma \eta f_{\boldsymbol{j}, \alpha}^{\dagger}\left[\sigma_{2}\right]_{\alpha \beta} f_{\boldsymbol{j}+\boldsymbol{e}_{x}+\sigma \boldsymbol{e}_{y}, \beta}^{\dagger}\right) \\
& \left.+ \text { h.c. }]-\frac{1}{2} h_{\mathrm{eff}} f_{\boldsymbol{j}, \alpha}^{\dagger}\left[\sigma_{3}\right]_{\alpha \beta} f_{\boldsymbol{j}, \beta}\right\},
\end{aligned}
$$

where $\alpha_{1}$ denotes the uniform temporal gauge field that has a finite value in the $Z_{2}$ polar state (see Ref. 18). Or equivalently,

$$
\begin{aligned}
\mathcal{H}_{\text {polar }} & =\sum_{\boldsymbol{k} ; k_{y}>0} \boldsymbol{f}_{\boldsymbol{k}}^{\dagger}\left\{-\alpha_{1} \gamma_{2}-\frac{\left|J_{1}\right|}{2}\left(s_{x}+s_{y}\right)\left(D \gamma_{3}-E \gamma_{25}\right)\right. \\
& \left.-J_{2} \chi c_{x} c_{y} \gamma_{4}-J_{2} \eta s_{x} s_{y} \gamma_{2}+\frac{1}{2} h_{\mathrm{eff}} \gamma_{35}\right\} \boldsymbol{f}_{\boldsymbol{k}} .
\end{aligned}
$$

\section{C. $S U(2)$ chiral $p$-wave state}

In the $S U(2)$ chiral $p$-wave state, the $d$-vectors on the nearest neighbor $x$-link and that on the $y$-link are collinear with each other. One of the two acquires an additional phase factor $i$, compared with the other,

$$
\begin{array}{ll}
D_{\boldsymbol{i} \boldsymbol{j}, \mu} & =\left\{\begin{array}{cc}
D \delta_{\mu, 1} & \left(\boldsymbol{i}=\boldsymbol{j}+\boldsymbol{e}_{x}\right), \\
i D \delta_{\mu, 1} & \left(\boldsymbol{i}=\boldsymbol{j}+\boldsymbol{e}_{y}\right),
\end{array}\right. \\
E_{\boldsymbol{i j}, \mu} & =0 .
\end{array}
$$

Eqs. 18a-b) hold under any magnetic field, provided that the $\boldsymbol{d}$-vector is perpendicular to the field.
The antiferromagnetic exchange interaction supports the 'uniform-RVB' configuration of the singlet pairings,, 26

$$
\eta_{i j}=0, \quad \chi_{i j}=\chi
$$

for $\boldsymbol{i}=\boldsymbol{j}+\boldsymbol{e}_{x} \pm \boldsymbol{e}_{y}$. The mean-field Hamiltonian for the $S U(2)$ chiral $p$-wave state takes the following form,

$$
\begin{aligned}
& \mathcal{H}_{\text {chiral } p \text { wave }}=\sum_{\boldsymbol{j}}\left\{\left[-\frac{\left|J_{1}\right|}{4} D\left(f_{\boldsymbol{j}, \alpha}^{\dagger}\left[\sigma_{3}\right]_{\alpha \beta} f_{\boldsymbol{j}+\boldsymbol{e}_{x}, \beta}^{\dagger}\right.\right.\right. \\
& \left.+i f_{\boldsymbol{j}, \alpha}^{\dagger}\left[\sigma_{3}\right]_{\alpha \beta} f_{\boldsymbol{j}+\boldsymbol{e}_{y}, \beta}^{\dagger}\right)-\frac{J_{2}}{4} \chi \sum_{\sigma= \pm} f_{\boldsymbol{j}, \alpha}^{\dagger} f_{\boldsymbol{j}+\boldsymbol{e}_{x}+\sigma \boldsymbol{e}_{y}, \alpha} \\
& \left.+ \text { h.c. }]-\frac{1}{2} h_{\mathrm{eff}} f_{\boldsymbol{j}, \alpha}^{\dagger}\left[\sigma_{3}\right]_{\alpha \beta} f_{\boldsymbol{j}, \beta}\right\}
\end{aligned}
$$

or equivalently,

$$
\begin{aligned}
\mathcal{H}_{\text {chiral } p \text { wave }}= & \sum_{\boldsymbol{k} ; k_{y}>0} \boldsymbol{f}_{\boldsymbol{k}}^{\dagger}\left\{-\frac{\left|J_{1}\right|}{2} D\left(s_{x} \boldsymbol{\gamma}_{3}+s_{y} \gamma_{34}\right)\right. \\
& \left.-J_{2} \chi c_{x} c_{y} \boldsymbol{\gamma}_{4}+\frac{1}{2} h_{\mathrm{eff}} \boldsymbol{\gamma}_{35}\right\} \boldsymbol{f}_{\boldsymbol{k}}
\end{aligned}
$$

\section{Fully polarized state out of 'flat-band' states}

The 'flat-band' states have only spin-triplet pairings on the ferromagnetic bonds, while no spin-singlet pairing on the antiferromagnetic bonds. According to our previous work,, 18 this state achieves the best mean-field energy among others in the strongly ferromagnetic regime $\left(\left|J_{1}\right| \gg J_{2}\right)$. In the absence of the external field, the triplet pairings in the 'flat-band' state are most generally characterized by a $U(1)$ phase, $\theta$, three orthogonal unit vectors $\left\{\boldsymbol{n}_{1}, \boldsymbol{n}_{2}, \boldsymbol{n}_{3}\right\}$ in the spin space, and two orthogonal unit vectors $\left\{\boldsymbol{m}_{1}, \boldsymbol{m}_{2}\right\}$ in the gauge space as follows

$$
\begin{gathered}
\boldsymbol{D}_{\boldsymbol{i} \boldsymbol{j}}= \begin{cases}\cos \theta \boldsymbol{n}_{1}\left(m_{1,3}+i m_{1,2}\right) & \left(\boldsymbol{i}=\boldsymbol{j}+\boldsymbol{e}_{x}\right), \\
\sin \theta \boldsymbol{n}_{1}\left(m_{2,3}+i m_{2,2}\right) & \left(\boldsymbol{i}=\boldsymbol{j}+\boldsymbol{e}_{y}\right),\end{cases} \\
\boldsymbol{E}_{\boldsymbol{i j}}= \begin{cases}\cos \theta\left(\boldsymbol{n}_{2}+i \boldsymbol{n}_{1} m_{1,1}\right) & \left(\boldsymbol{i}=\boldsymbol{j}+\boldsymbol{e}_{x}\right), \\
\sin \theta\left(\boldsymbol{n}_{3}+i \boldsymbol{n}_{1} m_{2,1}\right) & \left(\boldsymbol{i}=\boldsymbol{j}+\boldsymbol{e}_{y}\right),\end{cases}
\end{gathered}
$$

where

$$
\begin{aligned}
& \boldsymbol{n}_{1} \cdot \boldsymbol{n}_{2}=\boldsymbol{n}_{2} \cdot \boldsymbol{n}_{3}=\boldsymbol{n}_{3} \cdot \boldsymbol{n}_{1}=0 \\
& \boldsymbol{m}_{1} \cdot \boldsymbol{m}_{2}=0
\end{aligned}
$$

The energy dispersion of the Bogoliubov particle comprises two bands, which are totally flat in the momentum space and energetically separated by $2\left|J_{1}\right|$ from each other. In the remaining part of this section, we will argue that this state actually reduces to a fully polarized ferromagnetic state, once an infinitesimally small Zeeman field is applied.

Under the Zeeman field, it is energetically favorable that all the $d$-vectors are perpendicular to the field. To 
make this compatible with the orthogonality condition Eq. (23), the $U(1)$ phase $\theta$ is going to be locked in $\theta=\frac{\pi}{2} l$ with $l \in \mathbb{Z}$. Namely, when $\theta= \pm \frac{\pi}{2}$, only $\boldsymbol{n}_{3}$ and $\boldsymbol{n}_{1}$ are required to be perpendicular to the field, while, in the case of $\theta=0$ or $\pi$, only $\boldsymbol{n}_{1}$ and $\boldsymbol{n}_{2}$ are perpendicular to the field. Thereby, this locking reduces the 'flat-band' state into a decoupled one-dimensional fermion states running along either $x$-link or $y$-link. For example, when $\theta=0$, one of the flat-band states under an infinitesimally small field can be described with $\boldsymbol{n}_{1}=(1,0,0)$, $\boldsymbol{n}_{2}=(0,1,0)$, and $\boldsymbol{m}_{1}=(1,0,0)$. The corresponding $\mathrm{BdG}$ Hamiltonian is given as

$$
\begin{aligned}
\mathcal{H}_{\text {flat }} & =\sum_{j}\left\{\left[-\frac{\left|J_{1}\right|}{4}\left(i f_{\boldsymbol{j}, \alpha}^{\dagger}\left[\sigma_{1}\right]_{\alpha \beta} f_{\boldsymbol{j}+e_{x}, \beta}\right.\right.\right. \\
& \left.\left.\left.+f_{\boldsymbol{j}, \alpha}^{\dagger}\left[\sigma_{2}\right]_{\alpha \beta} f_{\boldsymbol{j}+e_{x}, \beta}\right)+ \text { h.c. }\right]-\frac{1}{2} h_{\mathrm{eff}} f_{\boldsymbol{j}, \alpha}^{\dagger}\left[\sigma_{3}\right]_{\alpha \beta} f_{\boldsymbol{j}, \beta}\right\} .
\end{aligned}
$$

Or equivalently,

$$
\begin{gathered}
\mathcal{H}_{\text {flat }}=\sum_{j_{y}=1}^{L_{y}} \sum_{-\pi<k_{x}<\pi} \boldsymbol{f}_{k_{x}, j_{y}}^{\dagger}\left\{-\frac{\left|J_{1}\right|}{2}\left(c_{x} \gamma_{31}+s_{x} \gamma_{23}\right)\right. \\
\left.+\frac{1}{2} h_{\mathrm{eff}} \gamma_{35}\right\} \boldsymbol{f}_{k_{x}, j_{y}}
\end{gathered}
$$

where $\boldsymbol{f}_{k_{x}, j_{y}}^{\dagger} \equiv\left(f_{k_{x}, j_{y}, \uparrow}^{\dagger}, f_{k_{x}, j_{y}, \downarrow}^{\dagger}, f_{-k_{x}, j_{y}, \uparrow}, f_{-k_{x}, j_{y}, \downarrow}\right)$ and $f_{k_{x}, j_{y}, \alpha} \equiv \frac{1}{\sqrt{L_{x}}} \sum_{j_{x}=1}^{L_{x}} e^{i k_{x} j_{x}} f_{j, \alpha}$ with $L_{x} L_{y}=N$.

When projected into the spin Hilbert space, the ground-state wavefunction of Eq. (26) reduces to a fully polarized ferromagnetic state. To see this, note that it is given by a composite of decoupled one-dimensional fermionic states running along the $x$-link; $\left|\Psi_{\text {flat }}\right\rangle=$ $\prod_{j_{y}=1}^{L_{y}}\left|\Psi_{j_{y}}\right\rangle$. For every $j_{y}=1, \cdots, L_{y},\left|\Psi_{j_{y}}\right\rangle$ is given by

$$
\begin{aligned}
\left|\Psi_{j_{y}}\right\rangle= & \prod_{-\pi<k_{x}<\pi}\left(-\cos \frac{\phi}{2} e^{i \frac{\pi}{4}+i \frac{k_{x}}{2}} f_{k_{x} \uparrow}^{\dagger}\right. \\
& \left.+\sin \frac{\phi}{2} e^{-i \frac{\pi}{4}-i \frac{k_{x}}{2}} f_{k_{x}, \downarrow}^{\dagger}\right)|0\rangle,
\end{aligned}
$$

where $|0\rangle$ denotes the vacuum state of the fermions. We have omitted the index ' $j_{y}$ ', $f_{k_{x}, j_{y}, \alpha}^{\dagger} \rightarrow f_{k_{x}, \alpha}^{\dagger}$, since the argument holds for each $j_{y}$ independently. Note also that the quantization axis of the spin was taken along the field. The angle $\phi$ is defined as

$$
\phi=\tan ^{-1}\left[\frac{\left|J_{1}\right|}{h_{\mathrm{eff}}}\right]
$$

in the range $-\frac{\pi}{2} \leq \phi \leq \frac{\pi}{2}$.

The inner-product between $\left|\Psi_{j_{y}}\right\rangle$ and an Ising spin configuration $\left|\left\{\sigma_{j_{x}}\right\}\right\rangle \equiv\left\{\prod_{j_{x}=1}^{L_{x}} f_{j_{x}, \sigma_{j_{x}}}^{\dagger}\right\}|0\rangle$ is given by a determinant of the $L_{x} \times L_{x}$ matrix,

$$
\left\langle\left\{\sigma_{j_{x}}\right\} \mid \Psi_{j_{y}}\right\rangle=\operatorname{det}\left[\begin{array}{cccc}
a_{\sigma_{1}} e^{i \frac{\pi \sigma_{1}}{4}} e^{i \frac{2 \pi}{L_{x}}\left(-1+\frac{\sigma_{1}}{2}\right)} & a_{\sigma_{2}} e^{i \frac{\pi \sigma_{2}}{4}} e^{i \frac{2 \pi}{L_{x}}\left(-2+\frac{\sigma_{2}}{2}\right)} & \cdots & a_{\sigma_{L_{x}}} e^{i \frac{\pi \sigma_{L_{x}}}{4}} e^{i \frac{2 \pi}{L_{x}}\left(-L_{x}+\frac{\sigma_{L_{x}}}{2}\right)} \\
a_{\sigma_{1}} e^{i \frac{\pi \sigma_{1}}{4}} e^{i \frac{4 \pi}{L_{x}}\left(-1+\frac{\sigma_{1}}{2}\right)} & a_{\sigma_{2}} e^{i \frac{\sigma_{2}}{4}} e^{i \frac{4 \pi}{L_{x}}\left(-2+\frac{\sigma_{2}}{2}\right)} & \cdots & a_{\sigma_{L_{x}}} e^{i \frac{\pi L_{x}}{4}} e^{i \frac{i \pi}{L_{x}}\left(-L_{x}+\frac{\sigma_{L_{x}}}{2}\right)} \\
\vdots & \vdots & \ddots & \vdots \\
a_{\sigma_{1}} e^{i \frac{\pi \sigma_{1}}{4}} e^{i 2 \pi\left(-1+\frac{\sigma_{1}}{2}\right)} & a_{\sigma_{2}} e^{i \frac{\pi \sigma_{2}}{4}} e^{i 2 \pi\left(-2+\frac{\sigma_{2}}{2}\right)} & \cdots & a_{\sigma_{L_{x}}} e^{i \frac{\pi \sigma_{L_{x}}}{4}} e^{i 2 \pi\left(-L_{x}+\frac{\sigma_{L_{x}}}{2}\right)}
\end{array}\right]
$$

where $\sigma= \pm 1$ ( $\uparrow, \downarrow$ respectively) with $a_{\sigma} \equiv-\delta_{\sigma, 1} \cos \frac{\phi}{2}+$ $\delta_{\sigma,-1} \sin \frac{\phi}{2}$. This determinant becomes non-zero, if and only if all the spins are pointing upward or pointing downward: otherwise, the $L_{x} \times L_{x}$ matrix always has two adjacent column-vectors which are parallel to each other in the $L_{x}$ dimensional space, at a domain wall with $\left(\sigma_{l}, \sigma_{l+1}\right)=(-1,1)$. Accordingly, we have

$$
\begin{aligned}
\left\langle\left\{\sigma_{j_{x}}\right\} \mid \Psi_{j_{y}}\right\rangle= & \left(-\cos \frac{\phi}{2}\right)^{L_{x}} \prod_{j_{x}=1}^{L_{x}} \delta_{\sigma_{j_{x}},+1} \\
& +\left(\sin \frac{\phi}{2}\right)^{L_{x}} \prod_{j_{x}=1}^{L_{x}} \delta_{\sigma_{j_{x}},-1}
\end{aligned}
$$

Note that $\cos \frac{\phi}{2}>\sin \frac{\phi}{2} \geq 0$ for $h_{\text {eff }}>0$. This suggests that, when normalized in the thermodynamic limit,
Eq. (27) always reduces to the ferromagnetic state,

$$
\lim _{L_{x} \rightarrow \infty} \frac{1}{\sqrt{\left\langle\Psi_{j_{y}} \mid \Psi_{j_{y}}\right\rangle}}\left|\Psi_{j_{y}}\right\rangle=|\uparrow, \uparrow, \cdots, \uparrow\rangle
$$

for any $j_{y}=1, \cdots, L_{y}$.

\section{TRIAL MANY-BODY WAVEFUNCTION}

As shown in the previous section, the projected 'flatband' state under an infinitesimally small field reduces to the fully polarized ferromagnetic state. As such, we regard that the projected 'flat-band' state in the absence of the field is the trivial ferromagnetic state, whose energy is exactly estimated as $-\frac{1}{2}\left(\left|J_{1}\right|-J_{2}\right)$ (per site). Hence we will focus on the character and energetics of the other three spin-triplet RVB states, (i) $Z_{2}$ planar state, (ii) $Z_{2}$ 
polar state and (iii) $S U(2)$ chiral $p$-wave state. To this end, we will construct in this section the projected spintriplet BCS wavefunctions out of their respective meanfield Hamiltonians.

\section{A. Projected spin-triplet BCS wavefunctions}

Let us first derive a BCS 'many-body' wavefunction for the BdG Hamiltonian which has both spin-triplet and spin-singlet pairings and hopping integrals. Suppose that we have a mean-field Hamiltonian $\mathcal{H} \equiv \sum_{k_{y}>0} \boldsymbol{f}_{\boldsymbol{k}}^{\dagger} \boldsymbol{H}_{\boldsymbol{k}} \boldsymbol{f}_{\boldsymbol{k}}$ and a $4 \times 4$ matrix $\boldsymbol{H}_{\boldsymbol{k}}$ is diagonalized by a unitary transformation $\boldsymbol{U}_{\boldsymbol{k}}$. We typically use Eqs. (13/1721) for $\mathcal{H}$. For these Hamiltonians, the eigenvalues always appear in the particle-hole pairwise manner,

$$
\boldsymbol{H}_{\boldsymbol{k}} \boldsymbol{U}_{\boldsymbol{k}}=\boldsymbol{U}_{\boldsymbol{k}}\left[\begin{array}{llll}
\lambda_{\boldsymbol{k}, 1} & & & \\
& \lambda_{\boldsymbol{k}, 2} & & \\
& & -\lambda_{\boldsymbol{k}, 2} & \\
& & & -\lambda_{\boldsymbol{k}, 1}
\end{array}\right] .
$$

As such, without loss of generality, we can assume $\lambda_{\boldsymbol{k}, j}$ $(j=1,2)$ to be positive (semi-)definite. Defining the Bogoliubov particle $\gamma_{\boldsymbol{k}, j}^{(\dagger)}(j=1,2)$ as

$$
\begin{aligned}
& \left(\begin{array}{llll}
\gamma_{\boldsymbol{k}, 1}^{\dagger} & \gamma_{\boldsymbol{k}, 2}^{\dagger} & \gamma_{-\boldsymbol{k}, 2} & \gamma_{-\boldsymbol{k}, 1}
\end{array}\right) \\
& \equiv\left(\begin{array}{llll}
f_{\boldsymbol{k}, \uparrow}^{\dagger} & f_{\boldsymbol{k}, \downarrow}^{\dagger} & f_{-\boldsymbol{k}, \uparrow} & f_{-\boldsymbol{k}, \downarrow}
\end{array}\right) \boldsymbol{U}_{\boldsymbol{k}},
\end{aligned}
$$

we obtain

$$
\mathcal{H}=\sum_{j=1}^{2} \sum_{\boldsymbol{k} ; k_{y}>0}\left\{\lambda_{\boldsymbol{k}, j} \gamma_{\boldsymbol{k}, j}^{\dagger} \gamma_{\boldsymbol{k}, j}-\lambda_{\boldsymbol{k}, j} \gamma_{-\boldsymbol{k}, j} \gamma_{-\boldsymbol{k}, j}^{\dagger}\right\} .
$$

Since $\lambda_{\boldsymbol{k}, j} \geq 0$, the mean-field ground state wavefunction $\mid$ g.s. $\rangle$ is a vacuum of the Bogoliubov particles, i.e., $\gamma_{\boldsymbol{k}, j} \mid$ g.s. $\rangle=\gamma_{-\boldsymbol{k}, j} \mid$ g.s. $\rangle=0$ for any $\boldsymbol{k}$ and $j$, which leads to

$$
\mid \text { g.s. }\rangle \propto \prod_{\boldsymbol{k} ; k_{y}>0}\left\{\gamma_{\boldsymbol{k}, 1} \gamma_{\boldsymbol{k}, 2} \gamma_{-\boldsymbol{k}, 2} \gamma_{-\boldsymbol{k}, 1}\right\}|0\rangle .
$$

Substituting Eq. (31) into Eq. (33), one can easily obtain

$$
\begin{gathered}
\prod_{\boldsymbol{k} ; k_{y}>0}\left\{\gamma_{\boldsymbol{k}, 1} \gamma_{\boldsymbol{k}, 2} \gamma_{-\boldsymbol{k}, 2} \gamma_{-\boldsymbol{k}, 1}\right\}|0\rangle \\
=c \prod_{\boldsymbol{k} ; k_{y}>0}\left\{1+a_{\boldsymbol{k}} f_{-\boldsymbol{k}, \uparrow}^{\dagger} f_{\boldsymbol{k}, \downarrow}^{\dagger}+b_{\boldsymbol{k}} f_{-\boldsymbol{k}, \downarrow}^{\dagger} f_{\boldsymbol{k}, \downarrow}^{\dagger}\right. \\
\left.+a_{\boldsymbol{k}}^{\prime} f_{-\boldsymbol{k}, \uparrow}^{\dagger} f_{\boldsymbol{k}, \uparrow}^{\dagger}+b_{\boldsymbol{k}}^{\prime} f_{-\boldsymbol{k}, \downarrow}^{\dagger} f_{\boldsymbol{k}, \uparrow}^{\dagger}+c_{\boldsymbol{k}} f_{-\boldsymbol{k}, \uparrow}^{\dagger} f_{-\boldsymbol{k}, \downarrow}^{\dagger} f_{\boldsymbol{k}, \uparrow}^{\dagger} f_{\boldsymbol{k}, \downarrow}^{\dagger}\right\}|0\rangle
\end{gathered}
$$

with

$$
\begin{aligned}
c= & \prod_{k_{y}>0}\left\{-\left[\boldsymbol{U}_{\boldsymbol{k}}\right]_{1,3}\left[\boldsymbol{U}_{\boldsymbol{k}}\right]_{2,4}+\left[\boldsymbol{U}_{\boldsymbol{k}}\right]_{2,3}\left[\boldsymbol{U}_{\boldsymbol{k}}\right]_{1,4}\right\} \\
& \times\left\{\left[\boldsymbol{U}_{\boldsymbol{k}}\right]_{1,1}^{*}\left[\boldsymbol{U}_{\boldsymbol{k}}\right]_{2,2}^{*}-\left[\boldsymbol{U}_{\boldsymbol{k}}\right]_{1,2}^{*}\left[\boldsymbol{U}_{\boldsymbol{k}}\right]_{2,1}^{*}\right\}
\end{aligned}
$$

and

$$
\begin{aligned}
a_{\boldsymbol{k}} & =\frac{\left[\boldsymbol{U}_{\boldsymbol{k}}\right]_{1,1}^{*}\left[\boldsymbol{U}_{\boldsymbol{k}}\right]_{3,2}^{*}-\left[\boldsymbol{U}_{\boldsymbol{k}}\right]_{1,2}^{*}\left[\boldsymbol{U}_{\boldsymbol{k}}\right]_{3,1}^{*}}{\left[\boldsymbol{U}_{\boldsymbol{k}}\right]_{1,1}^{*}\left[\boldsymbol{U}_{\boldsymbol{k}}\right]_{2,2}^{*}-\left[\boldsymbol{U}_{\boldsymbol{k}}\right]_{1,2}^{*}\left[\boldsymbol{U}_{\boldsymbol{k}}\right]_{2,1}^{*}}, \\
b_{\boldsymbol{k}} & =\frac{\left[\boldsymbol{U}_{\boldsymbol{k}}\right]_{1,1}^{*}\left[\boldsymbol{U}_{\boldsymbol{k}}\right]_{4,2}^{*}-\left[\boldsymbol{U}_{\boldsymbol{k}}\right]_{1,2}^{*}\left[\boldsymbol{U}_{\boldsymbol{k}}\right]_{4,1}^{*}}{\left[\boldsymbol{U}_{\boldsymbol{k}}\right]_{1,1}^{*}\left[\boldsymbol{U}_{\boldsymbol{k}}\right]_{2,2}^{*}-\left[\boldsymbol{U}_{\boldsymbol{k}}\right]_{1,2}^{*}\left[\boldsymbol{U}_{\boldsymbol{k}}\right]_{2,1}^{*},} \\
a_{\boldsymbol{k}}^{\prime} & =-\frac{\left[\boldsymbol{U}_{\boldsymbol{k}}\right]_{2,1}^{*}\left[\boldsymbol{U}_{\boldsymbol{k}}\right]_{3,2}^{*}-\left[\boldsymbol{U}_{\boldsymbol{k}}\right]_{2,2}^{*}\left[\boldsymbol{U}_{\boldsymbol{k}}\right]_{3,1}^{*}}{\left[\boldsymbol{U}_{\boldsymbol{k}}\right]_{1,1}^{*}\left[\boldsymbol{U}_{\boldsymbol{k}}\right]_{2,2}^{*}-\left[\boldsymbol{U}_{\boldsymbol{k}}\right]_{1,2}^{*}\left[\boldsymbol{U}_{\boldsymbol{k}}\right]_{2,1}^{*}}, \\
b_{\boldsymbol{k}}^{\prime} & =-\frac{\left[\boldsymbol{U}_{\boldsymbol{k}}\right]_{2,1}^{*}\left[\boldsymbol{U}_{\boldsymbol{k}}\right]_{4,2}^{*}-\left[\boldsymbol{U}_{\boldsymbol{k}}\right]_{2,2}^{*}\left[\boldsymbol{U}_{\boldsymbol{k}}\right]_{4,1}^{*}}{\left[\boldsymbol{U}_{\boldsymbol{k}}\right]_{1,1}^{*}\left[\boldsymbol{U}_{\boldsymbol{k}}\right]_{2,2}^{*}-\left[\boldsymbol{U}_{\boldsymbol{k}}\right]_{1,2}^{*}\left[\boldsymbol{U}_{\boldsymbol{k}}\right]_{2,1}^{*}}, \\
c_{\boldsymbol{k}} & =-\frac{\left[\boldsymbol{U}_{\boldsymbol{k}}\right]_{3,1}^{*}\left[\boldsymbol{U}_{\boldsymbol{k}}\right]_{4,2}^{*}-\left[\boldsymbol{U}_{\boldsymbol{k}}\right]_{3,2}^{*}\left[\boldsymbol{U}_{\boldsymbol{k}}\right]_{4,1}^{*}}{\left[\boldsymbol{U}_{\boldsymbol{k}}\right]_{1,1}^{*}\left[\boldsymbol{U}_{\boldsymbol{k}}\right]_{2,2}^{*}-\left[\boldsymbol{U}_{\boldsymbol{k}}\right]_{1,2}^{*}\left[\boldsymbol{U}_{\boldsymbol{k}}\right]_{2,1}^{*}} .
\end{aligned}
$$

Notice that $c_{\boldsymbol{k}}=a_{\boldsymbol{k}} b_{\boldsymbol{k}}^{\prime}-a_{\boldsymbol{k}}^{\prime} b_{\boldsymbol{k}}$. This makes it possible to exponentiate the right hand side of Eq. (34) as

$$
\mid \text { g.s. }\rangle \equiv \exp \left[\sum_{\boldsymbol{k} ; k_{y}>0}\left[\boldsymbol{t}_{\boldsymbol{k}}\right]_{\alpha \beta} f_{-\boldsymbol{k}, \alpha}^{\dagger} f_{\boldsymbol{k}, \beta}^{\dagger}\right]|0\rangle
$$

with

$$
t_{k} \equiv\left[\begin{array}{ll}
a_{k}^{\prime} & a_{k} \\
b_{k}^{\prime} & b_{k}
\end{array}\right]
$$

Equation (37) generally has a finite weight not only on physical (i.e. spin) Hilbert space but also on those fermionic states having either double occupancy on a single site or an empty site. To obtain a variational manybody wavefunction in the physical spin Hilbert space, we need to project out these unphysical fermionic states, imposing 'single-fermion condition' on every site;

$$
\left.\left|\Psi_{\boldsymbol{\alpha}}\right\rangle \equiv \mathcal{P} \mid \text { g.s. }\right\rangle,
$$

where $\mathcal{P}$ stands for the projection operator onto the physical spin Hilbert space. The projected BCS wavefunction $\left|\Psi_{\boldsymbol{\alpha}}\right\rangle$ depends on the pairing and hopping fields encoded in the BdG Hamiltonian, such as $D, E, \chi, \eta$ and $h_{\mathrm{eff}}$. The characteristic of the mean fields is symbolically represented by the subscript $\boldsymbol{\alpha}$.

Within the spin Hilbert space, the wavefunction is expressed by its inner-product with an Ising spin configuration, $\left|\left\{\sigma_{j}\right\}\right\rangle=\left\{\prod_{j} f_{j, \sigma_{j}}^{\dagger}\right\}|0\rangle$. This product generally reduces to a Pfaffian, $, 32,35,36$

$$
\left\langle\left\{\sigma_{j}\right\} \mid \Psi_{\alpha}\right\rangle=\operatorname{Pf}\left[\boldsymbol{X}_{\alpha}\left(\left\{\sigma_{j}\right\}\right)\right],
$$

where $\boldsymbol{X}_{\alpha}\left(\left\{\sigma_{j}\right\}\right)$ denotes the $N \times N$ antisymmetric matrix given by 35,36

$$
\begin{aligned}
{\left[\boldsymbol{X}_{\alpha}\left(\left\{\sigma_{j}\right\}\right)\right]_{j, l} } & \equiv[\boldsymbol{t}(\boldsymbol{j}, \boldsymbol{l})]_{\sigma_{j}, \sigma_{l}}-[\boldsymbol{t}(\boldsymbol{l}, \boldsymbol{j})]_{\sigma_{l}, \sigma_{j}}, \\
{[\boldsymbol{t}(\boldsymbol{j}, \boldsymbol{l})]_{\alpha, \beta} } & \equiv \frac{1}{N} \sum_{\boldsymbol{k} ; k_{y}>0} e^{i \boldsymbol{k} \cdot(\boldsymbol{j}-\boldsymbol{l})}\left[\boldsymbol{t}_{\boldsymbol{k}}\right]_{\alpha, \beta} .
\end{aligned}
$$

Note that the boundary condition for the momentum $\boldsymbol{k}$ remains arbitrary in Eq. (41). To fix this arbitrariness, let 
us require that the spin wavefunction given by Eq. (40) is an eigenstate of translations,

$$
\left\langle\left\{\sigma_{T_{a}(\boldsymbol{j})}\right\} \mid \Psi_{\alpha}\right\rangle=e^{i \theta_{a}}\left\langle\left\{\sigma_{\boldsymbol{j}}\right\} \mid \Psi_{\alpha}\right\rangle,
$$

where $T_{a}$ denotes the lattice translational operation by $\boldsymbol{e}_{a}$, i.e., $T_{a}(\boldsymbol{j})=\boldsymbol{j}+\boldsymbol{e}_{a}(a=x, y)$. In fact, the preceding exact diagonalization studies ${ }^{37}$ suggest that the states with non-zero $Q$ vectors are unlikely realized in any intermediate coupling regime of the present $J_{1^{-}}$ $J_{2}$ model, so that we impose the translational invariance $\left(e^{i \theta_{x}}, e^{i \theta_{y}}\right)=(1,1)$ on Eq. (42). To satisfy this requirement, the fermion's momenta in Eq. (41) have only to observe either the anti-periodic boundary condition (APBC), i.e., $k_{a}=\left(2 n_{a}-1\right) \pi / L_{a}$ with $n_{a}=$ $-L_{a} / 2+1, \cdots, L_{a} / 2$, or the periodic boundary condition (PBC), i.e., $k_{a}=2 n_{a} \pi / L_{a}$. For the two-dimensional models, the trial wavefunctions have four options. When both $k_{x}$ and $k_{y}$ satisfy the anti-periodic boundary condition, the total momentum carried by our trial spin wavefunction is indeed at the $\Gamma$-point, i.e. $\left(e^{i \theta_{x}}, e^{i \theta_{y}}\right)=(1,1)$; to see this, one has only to relate $\boldsymbol{X}_{\alpha}\left(\left\{\sigma_{T_{a}(\boldsymbol{j})}\right\}\right)$ with $\boldsymbol{X}_{\alpha}\left(\left\{\sigma_{j}\right\}\right)$ in terms of a certain elementary row/column operation $O_{a}$,

$$
\boldsymbol{X}_{\alpha}\left(\left\{\sigma_{T_{a}(\boldsymbol{j})}\right\}\right)=O_{a}^{T} \boldsymbol{X}_{\alpha}\left(\left\{\sigma_{j}\right\}\right) O_{a},
$$

where $O_{a}$ exchanges site indices of $X_{\alpha}\left(\left\{\sigma_{j}\right\}\right)$ according to the lattice translation operator $T_{a}$. Similarly, when $k_{x}$ satisfies the periodic boundary condition while $k_{y}$ does the anti-periodic boundary condition or vice versa, the momenta carried by the projected BCS wavefunction can be shown to be $\left(e^{i \theta_{x}}, e^{i \theta_{y}}\right)=\left((-1)^{\left(L_{y}-1\right) L_{x}}, 1\right)$ or $\left(e^{i \theta_{x}}, e^{i \theta_{y}}\right)=\left(1,(-1)^{\left(L_{x}-1\right) L_{y}}\right)$, respectively. In what follows, we only consider the systems with even length $L_{x}$ and $L_{y}$, to impose the translational invariance of the total wavefunction, i.e., $\left(e^{i \theta_{x}}, e^{i \theta_{y}}\right)=(1,1)$.

Notice also that, when both $k_{x}$ and $k_{y}$ observe the periodic boundary condition, any of the projected BCS wavefunctions derived from Eqs. (13]17/21) cannot be expressed in terms of a single Pfaffian. This is roughly because, being either $d$-wave or $p$-wave, all the pairing fields in the Cooper channel always vanish at the four time-reversal invariant momentums points $(0,0),(0, \pi)$, $(\pi, 0)$ and $(\pi, \pi)$, where a $4 \times 4 \mathrm{BdG}$ Hamiltonian reduces to a $2 \times 2$ Bloch Hamiltonian having no anomalous part. As a result, the state-basis representation of the projected BCS wavefunction becomes relatively cumbersome. In this paper, we study only those projected BCS wavefunctions derived based on the other three boundary conditions. Following the standard literature $\stackrel{1,24}{\longleftarrow}$ we name the projected BCS wavefunction defined with the $\mathrm{APBC}$ in the both direction as the "wavefunction in the $(\pi, \pi)$-topological sector' and that with the $\mathrm{PBC}$ in one direction and the APBC in the other as the "wavefunction in the $(0, \pi)$ or $(\pi, 0)$-topological sector.'

\section{B. Quantum spin number projection}

Our Hamiltonian has the global $S U(2)$ spin rotational symmetry, while the trial wavefunctions constructed from spin-triplet pairing states explicitly break this continuous symmetry by hand. Such a symmetry breaking is supposed to occur only in the thermodynamic limit. The ground-state wavefunction in a finite-size system can be always identified as an eigenstate of the symmetry groups of the Hamiltonian. Accordingly, it is naturally expected that the energy of the trial state will be further improved, when the state being projected onto the eigenspace of an appropriate quantum number associated with the spinrotational symmetries. Thus, we also consider as our trial state the projections of the spin-triplet BCS wavefunctions with the quantum spin numbers.

The spin projection operator which filters out a state with the total spin $S=L$ and the $z$-component of the total spin $S_{z}=M$ has a form ${ }^{38,39}$

$$
\begin{aligned}
\mathcal{P}_{S_{z}=M} \mathcal{P}_{S=L} \equiv & \frac{2 L+1}{8 \pi^{2}} \int_{0}^{2 \pi} d \alpha \int_{0}^{\pi} d \beta \sin \beta \int_{0}^{2 \pi} d \gamma \\
& \times P_{L}(\cos \beta) e^{i \alpha\left(\hat{S}_{z}-M\right)} e^{i \beta \hat{S}_{y}} e^{i \gamma \hat{S}_{z}}
\end{aligned}
$$

where $P_{L}$ denotes the $L$-th Legendre polynomial, and $\mathcal{P}_{S=L}$ and $\mathcal{P}_{S_{z}=M}$ denote the projection operators filtering out a state with the total spin $S=L$ and a state with the $z$-component of the total spin $S_{z}=M$, respectively.

Combining this with Eqs. (37)-(41), we obtain the projected BCS wavefunction with the quantum spin number projection as

$$
\begin{aligned}
& \left\langle\left\{\sigma_{\boldsymbol{j}}\right\}\left|\mathcal{P}_{S_{z}=M} \mathcal{P}_{S=L}\right| \Psi_{\boldsymbol{\alpha}}\right\rangle=\frac{2 L+1}{4 \pi} \times \\
& \int_{0}^{\pi} d \beta \sin \beta \int_{0}^{2 \pi} d \gamma P_{L}(\cos \beta) \operatorname{Pf}\left[\boldsymbol{X}_{\boldsymbol{\alpha}}\left(\left\{\sigma_{\boldsymbol{j}}\right\} ; \beta, \gamma\right)\right]
\end{aligned}
$$

under the condition $\frac{1}{2} \sum_{\boldsymbol{j}} \sigma_{\boldsymbol{j}}=M$. Here, the $N \times N$ antisymmetric matrix $\boldsymbol{X}_{\alpha}\left(\left\{\sigma_{j}\right\} ; \beta, \gamma\right)$ is defined the same as in Eq. (41) with the $2 \times 2$ matrix $\boldsymbol{t}(\boldsymbol{j}, \boldsymbol{l})$ being redefined in a rotated spin frame;

$$
\begin{aligned}
{\left[\boldsymbol{X}_{\alpha}\left(\left\{\sigma_{\boldsymbol{j}}\right\} ; \beta, \gamma\right)\right]_{\boldsymbol{j}, \boldsymbol{l}} \equiv } & {\left[\boldsymbol{V}_{\beta, \gamma} \boldsymbol{t}(\boldsymbol{j}, \boldsymbol{l}) \boldsymbol{V}_{\beta, \gamma}^{-1}\right]_{\sigma_{\boldsymbol{j}}, \sigma_{l}} } \\
& -\left[\boldsymbol{V}_{\beta, \gamma} \boldsymbol{t}(\boldsymbol{l}, \boldsymbol{j}) \boldsymbol{V}_{\beta, \gamma}^{-1}\right]_{\sigma_{l}, \sigma_{\boldsymbol{j}}}
\end{aligned}
$$

where

$$
\boldsymbol{V}_{\beta, \gamma} \equiv\left[\begin{array}{cc}
\cos \frac{\beta}{2} & -\sin \frac{\beta}{2} \\
\sin \frac{\beta}{2} & \cos \frac{\beta}{2}
\end{array}\right]\left[\begin{array}{cc}
e^{i \frac{\gamma}{2}} & 0 \\
0 & e^{-i \frac{\gamma}{2}}
\end{array}\right] .
$$

To integrate over $\beta$ and $\gamma$ numerically in Eq. (44), we employ the Gauss-Legendre quadrature. When projecting into the singlet space, i.e. $S=0$, with the system size $N=6 \times 6 \sim 12 \times 12$, we typically used $10 \sim 16$ mesh points for the integration over $\beta$ and $10 \sim 20$ mesh points for that of $\gamma \underline{\underline{40}}$ 


\section{ENERGY OPTIMIZATION AND ENERGETICS}

In this section, we optimize the energies of the projected (i) $Z_{2}$ planar state, (ii) $Z_{2}$ polar state and (iii) $S U(2)$ chiral $p$-wave state and compare their minimized energies with those of the ferromagnetic state and the collinear antiferromagnetic state. Specifically, we have numerically calculated the expectation values of the energy for these projected BCS wavefunctions, taking the quantum spin number projection onto the subspace with either $S_{z}=0$ or $S=0$;

$$
E_{\boldsymbol{\alpha}}^{S_{z}=0}=\frac{\left\langle\Psi_{\boldsymbol{\alpha}}\left|\mathcal{P}_{S_{z}=0} H \mathcal{P}_{S_{z}=0}\right| \Psi_{\boldsymbol{\alpha}}\right\rangle}{\left\langle\Psi_{\boldsymbol{\alpha}}\left|\mathcal{P}_{S_{z}=0}\right| \Psi_{\boldsymbol{\alpha}}\right\rangle}
$$

and

$$
E_{\boldsymbol{\alpha}}^{S=0}=\frac{\left\langle\Psi_{\boldsymbol{\alpha}}\left|\mathcal{P}_{S=0} H \mathcal{P}_{S=0}\right| \Psi_{\boldsymbol{\alpha}}\right\rangle}{\left\langle\Psi_{\boldsymbol{\alpha}}\left|\mathcal{P}_{S=0}\right| \Psi_{\boldsymbol{\alpha}}\right\rangle}
$$

We have further optimized these energies, tuning the variational parameters $\boldsymbol{\alpha}$ encoded in the original BCS wavefunctions, such as $D, E, \chi, \eta$ and $h_{\text {eff. }}$ For this optimization, we have employed the so-called stochastic reconfiguration method ${ }^{33.34}$

\section{A. Stochastic reconfiguration method}

Here we briefly review the stochastic reconfiguration (SR) method ${ }^{33,34}$ In this optimization method, a usual steepest descent (SD) method is modified in such a way that information of the 'quantum distance' between wavefunctions is included. The quantum distance is chosen to be the square distance between two normalized wavefunctions defined in two different parameter points, say $\boldsymbol{\alpha}$ and $\boldsymbol{\alpha}+\delta \boldsymbol{\alpha}$, in the form

$$
\Delta_{\mathrm{SR}}^{2} \equiv\left[\left\langle\bar{\Psi}_{\boldsymbol{\alpha}+\delta \boldsymbol{\alpha}}\left|-\left\langle\bar{\Psi}_{\boldsymbol{\alpha}}\right|\right]\left[\left|\bar{\Psi}_{\boldsymbol{\alpha}+\delta \boldsymbol{\alpha}}\right\rangle-\left|\bar{\Psi}_{\boldsymbol{\alpha}}\right\rangle\right]\right.\right.
$$

with

$$
\left|\bar{\Psi}_{\alpha}\right\rangle=\left|\Psi_{\alpha}\right\rangle\left\{\left\langle\Psi_{\alpha} \mid \Psi_{\alpha}\right\rangle\right\}^{-\frac{1}{2}} .
$$

Regarding $|\delta \alpha|$ as a small quantities, we can expand this quantum distance in terms of $\delta \boldsymbol{\alpha}$,

$$
\Delta_{\mathrm{SR}}^{2}=\sum_{j, m} \delta \alpha_{j} \delta \alpha_{m}\left[\boldsymbol{S}_{\alpha}\right]_{j, m}+\mathcal{O}\left(\delta \boldsymbol{\alpha}^{3}\right),
$$

where the metric tensor $\left[\boldsymbol{S}_{\alpha}\right]$ is defined in the variational parameter space as

$$
\left[\boldsymbol{S}_{\alpha}\right]_{j, m} \equiv\left\langle\partial_{\alpha_{j}} \bar{\Psi}_{\boldsymbol{\alpha}} \mid \partial_{\alpha_{m}} \bar{\Psi}_{\boldsymbol{\alpha}}\right\rangle+\text { c.c.. }
$$

In the standard steepest descent (SD) method, the variational parameters are changed along the gradient of an energy, $\delta \alpha_{j}=\lambda \partial_{\alpha_{j}} E_{\boldsymbol{\alpha}}$ with $E_{\boldsymbol{\alpha}}=\left\langle\bar{\Psi}_{\boldsymbol{\alpha}}|H| \bar{\Psi}_{\boldsymbol{\alpha}}\right\rangle$ and a small positive value $\lambda$. Meanwhile, the SR method determines the optimal direction, by minimizing the energy on the contour-(super)sphere of the equal quantum distance. A variational principle with constraint dictates that the optimal direction thus defined is given by $\delta \alpha_{j}=\lambda \sum_{m}\left[\boldsymbol{S}_{\alpha}^{-1}\right]_{j, m} \partial_{\alpha_{m}} E_{\boldsymbol{\alpha}}$. It is empirically recognized that the modification in terms of the metric tensor $\left[\boldsymbol{S}_{\alpha}\right]$ substantially improves the optimization efficiency, especially when the tensor has a highly non-flat structure in the variational parameter space $\underline{33,34}$

The numerical evaluation of the metric tensor and the gradient vector requires the summation over all the Ising spin configurations in the physical Hilbert space,

$$
\begin{array}{r}
{\left[\boldsymbol{S}_{\alpha}\right]_{m, n}=\frac{1}{2} \sum_{\left\{\sigma_{j}\right\}}\left(\mathcal{O}_{m,\left\{\sigma_{j}\right\}}^{*} \mathcal{O}_{n,\left\{\sigma_{j}\right\}}+\text { c.c. }\right) w_{\left\{\sigma_{j}\right\}}} \\
-\sum_{\left\{\sigma_{j}\right\}} \operatorname{Re} \mathcal{O}_{m,\left\{\sigma_{j}\right\}} w_{\left\{\sigma_{j}\right\}} \sum_{\left\{\sigma_{m}\right\}} \operatorname{Re} \mathcal{O}_{n,\left\{\sigma_{m}\right\}} w_{\left\{\sigma_{m}\right\}}, \\
\partial_{\alpha_{m}} E_{\boldsymbol{\alpha}}=\sum_{\left\{\sigma_{j}\right\}}\left(\mathcal{E}_{\left\{\sigma_{j}\right\}} \mathcal{O}_{m,\left\{\sigma_{j}\right\}}+\text { c.c. }\right) \omega_{\left\{\sigma_{j}\right\}} \\
-2 \sum_{\left\{\sigma_{j}\right\}} \operatorname{Re} \mathcal{O}_{m,\left\{\sigma_{j}\right\}} w_{\left\{\sigma_{j}\right\}} \sum_{\left\{\sigma_{m}\right\}} \operatorname{Re} \mathcal{E}_{\left\{\sigma_{m}\right\}} w_{\left\{\sigma_{m}\right\}},
\end{array}
$$

where

$$
\begin{aligned}
w_{\left\{\sigma_{j}\right\}} & =\frac{\left|\left\langle\left\{\sigma_{j}\right\} \mid \Psi_{\boldsymbol{\alpha}}\right\rangle\right|^{2}}{\left\langle\Psi_{\boldsymbol{\alpha}} \mid \Psi_{\boldsymbol{\alpha}}\right\rangle}, \\
\mathcal{O}_{m,\left\{\sigma_{j}\right\}} & =\frac{\left\langle\left\{\sigma_{j}\right\} \mid \partial_{\alpha_{m}} \Psi_{\boldsymbol{\alpha}}\right\rangle}{\left\langle\left\{\sigma_{\boldsymbol{j}}\right\} \mid \Psi_{\boldsymbol{\alpha}}\right\rangle}, \\
\mathcal{E}_{\left\{\sigma_{j}\right\}} & =\frac{\left\langle\Psi_{\boldsymbol{\alpha}}|H|\left\{\sigma_{j}\right\}\right\rangle}{\left\langle\Psi_{\boldsymbol{\alpha}} \mid\left\{\sigma_{\boldsymbol{j}}\right\}\right\rangle} .
\end{aligned}
$$

The SR method replaces this extensive summation by the statistical average where $w_{\left\{\sigma_{j}\right\}}$ is regarded as a probability density of the corresponding statistical ensemble. Specifically, we numerically create a Markov chain in which a binary configuration $\left\{\sigma_{j}\right\}$ is statistically generated with the probability $w_{\left\{\sigma_{j}\right\}}$. In the statistical ensemble thus defined, observables defined in Eqs. (54 55) are numerically evaluated;

$$
\begin{aligned}
& {\left[\boldsymbol{S}_{\boldsymbol{\alpha}}\right]_{m, n}=\frac{1}{2}\left(\overline{\mathcal{O}_{m} \mathcal{O}_{n}^{*}}+\text { c.c. }\right)-\frac{1}{4}\left(\overline{\mathcal{O}_{m}}+\text { c.c. }\right)\left(\overline{\mathcal{O}_{n}}+\text { c.c. }\right),} \\
& \partial_{\boldsymbol{\alpha}_{m}} E_{\boldsymbol{\alpha}}=\left(\overline{\mathcal{E} \mathcal{O}_{m}^{*}}+\text { c.c. }\right)-\frac{1}{2}(\overline{\mathcal{E}}+\text { c.c. })\left(\overline{\mathcal{O}_{m}}+\text { c.c. }\right) .
\end{aligned}
$$

To obtain the metric tensors and gradient vector, we usually take $1000 \sim 4000$ samplings per site. For a set of optimized variational parameters, we evaluate the energy (sec. IVB) and the correlation function (sec. VI), where we typically use $10^{5}$ samplings. As for the projected BCS wavefunction with the quantum spin number projection, one has only to replace $\left|\Psi_{\alpha}\right\rangle$ in Eqs. (553)-(55) by $\mathcal{P}_{S_{z}=M} \mathcal{P}_{S=L}\left|\Psi_{\boldsymbol{\alpha}}\right\rangle$. Those who are interested in the actual evaluation of these observables can consult Refs. 36 and 38 . 


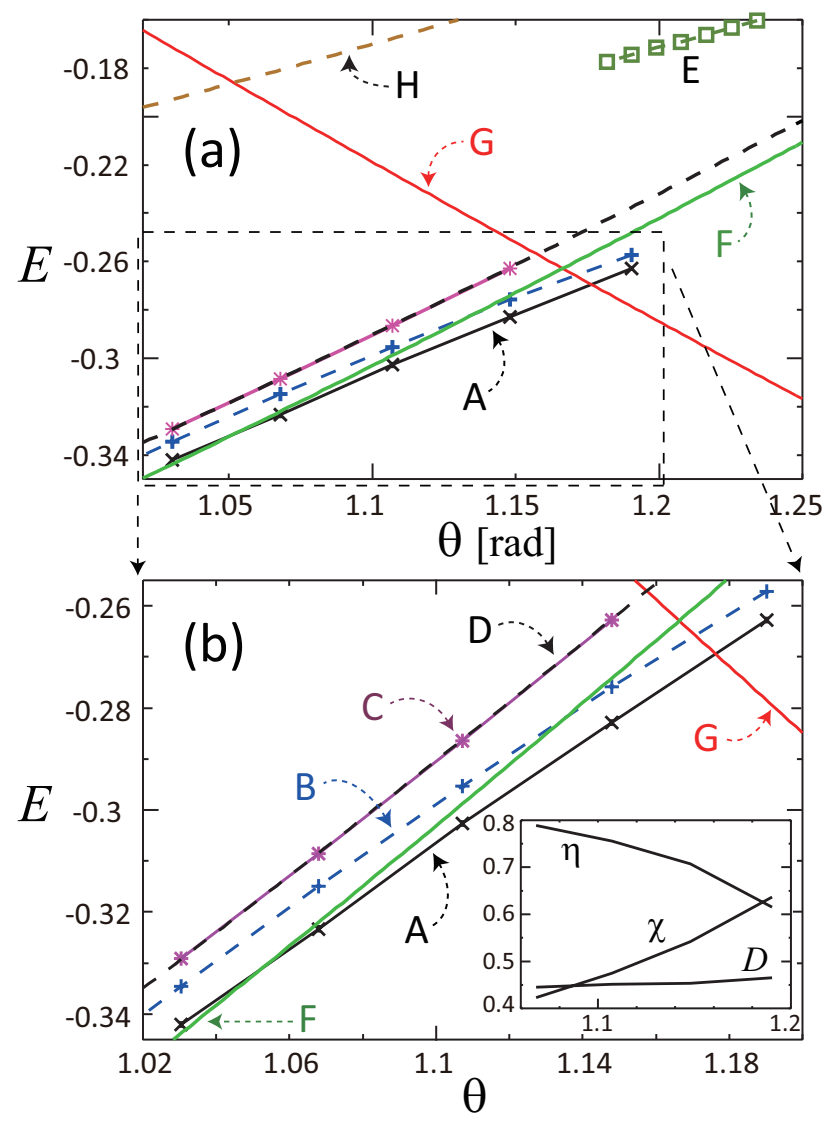

FIG. 3: (Color online) Energy comparison in the square lattice $J_{1}-J_{2}$ model as a function of $\theta$ with $\left(J_{1}, J_{2}\right)=$ $(-\sin \theta, \cos \theta)$. (a): Optimized energies of the planar states [(A) $\mathcal{P}_{S=0}\left|\Psi_{\text {planar }}\right\rangle$ and (B) $\left.\mathcal{P}_{S_{z}=0}\left|\Psi_{\text {planar }}\right\rangle\right]$, the polar state [(C) $\left.\mathcal{P}_{S_{z}=0}\left|\Psi_{\text {polar }}\right\rangle\right]$, the $\pi$-flux state [(D) $\left.\mathcal{P}_{S_{z}=0}\left|\Psi_{\pi \text {-flux }}\right\rangle\right]$, the $p$-wave chiral state $\left[(\mathrm{E}) \mathcal{P}_{S_{z}=0}\left|\Psi_{\text {chiral }}\right\rangle\right]$, and the collinear antiferromagnetic state $(\mathrm{F})$. The exact energies of the ferromagnetic state $(\mathrm{G})$ and the isolated dimer state $(\mathrm{H})$ are also shown. The projected state $\mathcal{P}_{S=0}\left|\Psi_{\text {planar }}\right\rangle$ is calculated in $8 \times 8$ spin system and $\mathcal{P}_{S_{z}=0}\left|\Psi_{\text {planar }}\right\rangle$ is in $10 \times 10$ spin system. (b): A part of figure (a) is enlarged. (inset): Optimized parameter values of $D, \chi$, and $\eta$ in $\mathcal{P}_{S=0}\left|\Psi_{\text {planar }}\right\rangle$ as a function of $\theta$. These values are rescaled, such that $\sqrt{D^{2}+\chi^{2}+\eta^{2}}=1$. The optimal values of the excitonic spin-triplet pairing field $E$ and the effective Zeeman field $h_{\text {eff }}$ are negligibly small.

\section{B. Energetics}

Figure 3 shows the optimized energies of the projected $Z_{2}$ planar states (both $\mathcal{P}_{S_{z}=0}\left|\Psi_{\text {planar }}\right\rangle$ and $\left.\mathcal{P}_{S=0}\left|\Psi_{\text {planar }}\right\rangle\right)$, the projected $Z_{2}$ polar state $\mathcal{P}_{S_{z}=0}\left|\Psi_{\text {polar }}\right\rangle$, and the projected $S U(2)$ chiral $p$-wave state $\mathcal{P}_{S_{z}=0}\left|\Psi_{\text {chiral }}\right\rangle$. We also compare these optimized energies with the exact energy of the fully polarized ferromagnetic state $E_{\text {ferro }}=-0.5\left(\left|J_{1}\right|-J_{2}\right)$, and the variationally optimized energies of the collinear antiferromagnetic state $\frac{42}{2} E_{\mathrm{CAF}}=-0.6682 J_{2}$ and the decoupled double $\pi$-flux state $\underline{\underline{41}} E_{\pi \text {-flux }}=-0.64 J_{2}$. The collinear antiferromagnetic state we considered in this

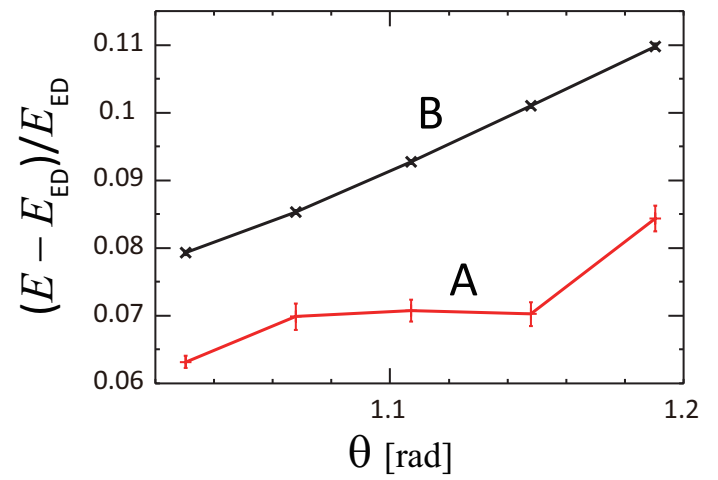

FIG. 4: (Color online) Comparison between the optimized energies of the projected planar states (A) $\mathcal{P}_{S=0}\left|\Psi_{\text {planar }}\right\rangle$ and (B) $\mathcal{P}_{S_{z}=0}\left|\Psi_{\text {planar }}\right\rangle$, and the ground state energy $E_{\mathrm{ED}}$ obtained by exact diagonalization, in the square lattice $J_{1}-J_{2}$ model, as a function of $\theta=\tan ^{-1}\left(-J_{1} / J_{2}\right)$. The system size is $6 \times 6$.

paper is composed by two 'decoupled' Néel-ordered states

$$
\left|\Psi_{\mathrm{CAF}}\right\rangle=\left|\Psi_{\mathrm{Neel}}\right\rangle_{\mathrm{A}}\left|\Psi_{\mathrm{Neel}}\right\rangle_{\mathrm{B}}
$$

each of which is defined on a non-frustrated square sublattice coupled with $J_{2}$ bonds, say A-sublattice or B-sublattice. For $\left|\Psi_{\text {Neel }}\right\rangle$, we employed the variational wavefunction numerically derived by Liang et.al.,$\frac{42}{4}$ which was maximally optimized on the antiferromagnetic square lattice in terms of the staggered magnetic moment, singlet pairing fields, and the Jastrow factor. Note that the expectation value of the ferromagnetic exchange interaction always vanishes in Eq. (56), though the spintriplet pairing fields connecting the two sublattices could possibly decrease the energy in general. In spite of this, however, the energy of Eq. (56) achieves about 94.5\% of the exact ground state energy in 36 spin cluster at $\left|J_{1}\right|=J_{2}$, whereas it achieves about $96.3 \%$ at $J_{1}=0$. We thus regard that, even in the presence of considerable $J_{1}$, Eq. (56) still gives an appropriate energetics for the collinear antiferromagnetic state of the $J_{1}-J_{2}$ model.

The energy comparison in Fig. 3 shows that the projected $Z_{2}$ planar state has the lowest energy in a finite range of the intermediate coupling regime, $0.417\left|J_{1}\right| \lesssim$ $J_{2} \lesssim 0.57\left|J_{1}\right|$, whereas the ferromagnetic state is the most stable in the strong $J_{1}$ regime, $J_{2} \lesssim 0.417\left|J_{1}\right|$, and the collinear antiferromagnetic state is in the strong $J_{2}$ regime, $0.57\left|J_{1}\right| \lesssim J_{2}$. The optimal energy of the planar state projected onto the $S_{z}=0$ sector in the $6 \times 6$ system achieves roughly $92 \% \sim 89 \%$ of the exact ground state energy obtained by the numerical diagonalization with the same system size. The energy becomes further decreased by $2 \% \sim 3 \%$, when the wavefunction is projected onto the $S=0$ space (see Fig. (4).

Figure 3 suggests that, contrary to the mean-field analysis, the projected chiral $p$-wave state hardly realizes in any of the intermediate coupling regime of the $J_{1}-J_{2}$ model, at least when the system size is $2 n \times 2 n$ 


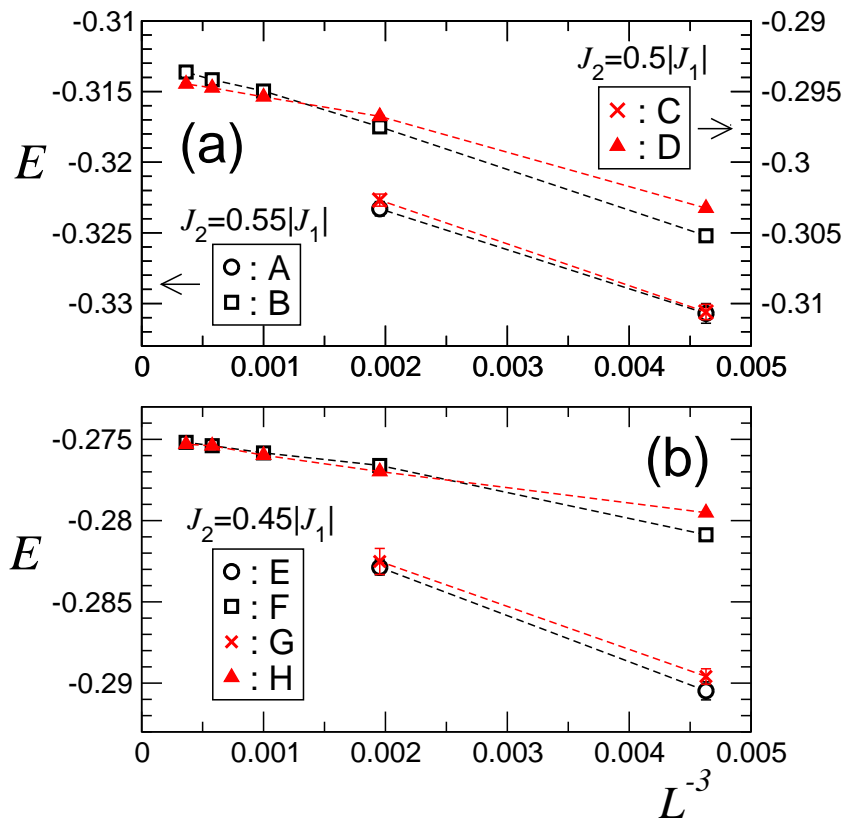

FIG. 5: (Color online) Size dependence of the optimized energies per site for the projected planar states in the $L \times L$ square lattice $J_{1}-J_{2}$ model with $J_{2}=0.55\left|J_{1}\right|$ (a: black dashed lines), $J_{2}=0.5\left|J_{1}\right|$ (a: red dashed lines), and $J_{2}=0.45\left|J_{1}\right|$ (b). The energy unit is taken to be $\sqrt{J_{1}^{2}+J_{2}^{2}}$. The projected states are taken as $\mathcal{P}_{S_{z}=0}\left|\Psi_{\text {planar }}\right\rangle(L=6 \sim 14)$ for the data $(\mathrm{B}, \mathrm{D}$, F, H) and as $\mathcal{P}_{S=0}\left|\Psi_{\text {planar }}\right\rangle(L=6,8)$ for the data $(\mathrm{A}, \mathrm{C}, \mathrm{E}$, $\mathrm{G})$. The horizontal axis is taken as $L^{-3}$. For $\mathcal{P}_{S_{z}=0}\left|\Psi_{\text {planar }}\right\rangle$ with $L=14$, we used the same variational parameters as those of $\mathcal{P}_{S_{z}=0}\left|\Psi_{\text {planar }}\right\rangle$ with $L=12$. For $\mathcal{P}_{S=0}\left|\Psi_{\text {planar }}\right\rangle$ with $L=8$, we used the same variational parameters as those of $\mathcal{P}_{S=0}\left|\Psi_{\text {planar }}\right\rangle$ with $L=6$. The data $\mathrm{G}$ and $\mathrm{H}$ are calculated in the $(0, \pi)$-topological sector and the others are in the $(\pi, \pi)$-topological sector.

$(n=3,4,5 \cdots)$. Moreover, the estimated energy of $\mathcal{P}_{S_{z}=0}\left|\Psi_{\text {chiral }}\right\rangle$ is already $30 \%$ higher than those of the ferromagnetic state and the projected $Z_{2}$ planar state, so that the situation is unlikely reversed, even when the wavefunction is further projected into the singlet space, i.e. $\mathcal{P}_{S=0}\left|\Psi_{\text {chiral }}\right\rangle$. Figure 3 also indicates that the $Z_{2}$ polar state is almost energetically degenerate with the double $\pi$-flux state, which indicates that the spin-triplet pairing does not lower the energy efficiently in this state. Indeed, we observed that the optimized value of the triplet pairing field in the polar state is less than $10 \%$ of the root square sum of all the variational parameters.

We also show the system size dependence of the optimized energy per site for the projected planar states in Fig. 5. The data for the state $\mathcal{P}_{S_{z}=0}\left|\Psi_{\text {planar }}\right\rangle$ indicates that the energy has a finite-size correction in the form $E(L) / L^{2}=\epsilon_{0}-c / L^{3}$. This observation is consistent with the existence of gapless Goldstone modes in the $Z_{2}$ planar state. Using the random phase approximation, we can show that the low energy dispersions of these gapless modes are always linear in the momentum, $\underline{\underline{43}}$ which suggests that the finite-size correction to the ground state energy per site decays in the form $-c / L^{3}$, the same as that of the two-dimensional antiferromagnetic Heisenberg model $\underline{\underline{44}}$

\section{V. $d$-WAVE SPIN-NEMATIC CHARACTER OF PROJECTED $Z_{2}$ PLANAR STATES}

In this section, we show that all the projected spintriplet RVB states derived from Eqs. (13]17/21) generally have the 'spin-nematic' properties; ordering of quadrupole moments without spontaneous ordering of magnetic dipole moments. In particular, we argue that the projected $Z_{2}$ planar state has a ' $d$-wave' spin-nematic character, or an ' $d$-wave' quadrupolar order, which is consistent with the nature of the spin nematic phase suggested by the exact diagonalization study ${ }^{9}$. All symmetries of the Anderson tower of spin nematic states are clarified from the decomposition of the $Z_{2}$ planar state.

To see the 'spin-nematic' character, notice first that all the mean-field states discussed in Sec. [I] are invariant under the spin $\pi$-rotation about the 3 -axis. Namely, spin-triplet $d$-vectors in these states are always lying in a plane perpendicular to the field (see Fig. 1), so that the spin $\pi$-rotation around the field changes the sign of the triplet pairing fields on the nearest-neighbor ferromagnetic bonds, while leaves intact the singlet pairing fields on the next-nearest-neighbor antiferromagnetic bonds. This sign change can be readily set off by the staggered gauge transformation $f_{j}^{\dagger} \rightarrow f_{j}^{\dagger}(-1)^{j_{x}+j_{y}}$. The whole unitary transformation is expressed as

$$
\begin{aligned}
U=\exp & {\left[i \pi \sum_{j, \sigma}\left(j_{x}+j_{y}\right) f_{j, \sigma}^{\dagger} f_{j, \sigma}\right] } \\
\times & \exp \left[i \frac{\pi}{2} \sum_{j} f_{j, \alpha}^{\dagger}\left[\sigma_{3}\right]_{\alpha \beta} f_{j, \beta}\right] .
\end{aligned}
$$

Since the mean-field Hamiltonian is invariant under this transformation, the transformed state $U \mid$ g.s. $\rangle$ is energetically degenerate with the original ground state $\mid$ g.s. $\rangle$. On the one hand, being a vacuum state of the Bogoliubov particle, the ground state should be unique, which leads to $U \mid$ g.s. $\rangle=\mathrm{e}^{\mathrm{i} \theta} \mid$ g.s. $\rangle$. Thereby, the projected BCS wavefunction generally satisfies the following relation

$$
\left\langle\left\{\sigma_{j}\right\} \mid \Psi_{\boldsymbol{\alpha}}\right\rangle=\left\langle\left\{\sigma_{j}\right\}|\mathcal{P}| \text { g.s. }\right\rangle=e^{-i \theta}\left\langle\left\{\sigma_{j}\right\}|\mathcal{P} U| \text { g.s. }\right\rangle \text {. }
$$

Since the unitary operator $U$ commutes with the projection $\mathcal{P}$, the right hand side can be further written as follows with the eigenvalues $S_{z} \equiv \frac{1}{2} \sum_{j} \sigma_{j}$,

$$
\left\langle\left\{\sigma_{j}\right\} \mid \Psi_{\boldsymbol{\alpha}}\right\rangle=e^{-i \theta} e^{i \pi S_{z}}\left\langle\left\{\sigma_{\boldsymbol{j}}\right\} \mid \Psi_{\boldsymbol{\alpha}}\right\rangle .
$$

One can easily fix the $U(1)$ phase factor, evaluating the product between the projected BCS wavefunctions and a fully polarized state $\left|\left\{\sigma_{j}=1\right\}\right\rangle \equiv\left\{\prod_{j} f_{j, \uparrow}^{\dagger}\right\}|0\rangle$,

$$
\left|\left\langle\left\{\sigma_{j}=1\right\} \mid \Psi_{\boldsymbol{\alpha}}\right\rangle\right|^{2}=\prod_{\boldsymbol{k} ; k_{y}>0}\left|a_{\boldsymbol{k}}^{\prime}\right|^{2},
$$


where $a_{\boldsymbol{k}}^{\prime}$ is defined in Eq. (36). Supposing that $a_{\boldsymbol{k}}^{\prime}$ is nonvanishing at any discretized momentum point at $k_{y}>0$, which actually holds true for the $Z_{2}$ planar state in the zero field case, i.e. Eq. (11), the projected BCS wavefunction has a finite weight in the eigenspace of $S_{z}=\frac{N}{2}$, $\left|\left\langle\left\{\sigma_{j}=1\right\} \mid \Psi_{\boldsymbol{\alpha}}\right\rangle\right| \neq 0$. To make this observation compatible with Eq. (59), the phase factor must take a form,

$$
e^{-i \theta}=(-1)^{\frac{N}{2}} \text {. }
$$

In general, we can prove Eqs. (59-60) more directly, only by imposing the spin- $\pi$ rotational symmetry onto the eigenvectors of a given Bogoliubov Hamiltonian.

Equation (59) guarantees the 'spin-nematic' character of the spin-triplet RVB states. Namely, when combined with Eq. (60), this identity requires that the wavefunctions have a finite weight only in the subspace with an even-integer $S_{z}$ for $N=4 l(l=1,2, \cdots)$ spin systems, whereas only in the subspace with an odd-integer $S_{z}$ for $N=4 l+2(l=0,1, \cdots)$ spin systems. Thus, the transverse local magnetization always vanishes in these projected spin-triplet RVB states,

$$
\left\langle\Psi_{\boldsymbol{\alpha}}\left|S_{j, \pm}\right| \Psi_{\boldsymbol{\alpha}}\right\rangle=0
$$

while the spin quadrupole moments in the transverse plane are allowed to have a finite value,

$$
\left\langle\Psi_{\boldsymbol{\alpha}}\left|S_{\boldsymbol{j},+} S_{\boldsymbol{m},+}\right| \Psi_{\boldsymbol{\alpha}}\right\rangle \equiv f(\boldsymbol{j}-\boldsymbol{m}) \neq 0,
$$

where $S_{j,+} S_{\boldsymbol{m},+}$ relates to the spin nematic operators in the form ${ }^{9} S_{\boldsymbol{j},+} S_{\boldsymbol{m},+}=\left(K_{\boldsymbol{j}, \boldsymbol{m}}^{11}-K_{\boldsymbol{j}, \boldsymbol{m}}^{22}\right)+2 i K_{\boldsymbol{j}, \boldsymbol{m}}^{12}$.

As for the $Z_{2}$ planar state derived from Eqs. (11]13), this quadrupole moments obey the $d$-wave spatial configuration,

$$
f\left(R_{\frac{\pi}{2}}(\boldsymbol{j}-\boldsymbol{m})\right)=-f(\boldsymbol{j}-\boldsymbol{m}),
$$

where $R_{\theta}$ denotes the space $\theta$-rotation around the axis perpendicular to the square-lattice plane. This $d$-wave nature comes from the fact that the planar state is invariant under the space $\frac{\pi}{2}$-rotation accompanied by the spin $\frac{\pi}{2}$-rotation around the 3 -axis (around the field) and the gauge transformation $f_{j, \sigma}^{\dagger} \rightarrow i(-1)^{j_{x}+j_{y}} f_{\boldsymbol{j}, \sigma}^{\dagger}$. Namely, the state is invariant under the following unitary transformation,

$$
\begin{aligned}
U^{\prime}=\exp & {\left[i \pi \sum_{j, \sigma}\left(j_{x}+j_{y}+\frac{1}{2}\right) f_{j, \sigma}^{\dagger} f_{j, \sigma}\right] } \\
\times & \exp \left[i \frac{\pi}{4} \sum_{j} f_{j, \alpha}^{\dagger}\left[\sigma_{3}\right]_{\alpha \beta} f_{j, \beta}\right] R_{\frac{\pi}{2}} .
\end{aligned}
$$

Utilizing this symmetry in the same way as we did for the spin $\pi$-rotation above, one can derive the following identity for the projected $Z_{2}$ planar state,

$$
\left\langle\left\{\sigma_{j}\right\} \mid \Psi_{\text {planar }}\right\rangle=(-1)^{\frac{N}{4}} e^{i \frac{\pi}{2} S_{z}}\left\langle\left\{\sigma_{R_{\frac{\pi}{2}}(j)}\right\} \mid \Psi_{\text {planar }}\right\rangle .
$$

TABLE I: Indices of the projected $Z_{2}$ planar states under the point group symmetries of the square lattice, where $N$ (multiples of 4) denotes the total number of the lattice points.

\begin{tabular}{cc}
\hline \hline point group symmetries & $\mathcal{P}_{S_{z}=2 n}\left|\Psi_{\text {planar }}\right\rangle$ \\
\hline time-reversal (for $n=0$ and zero-field case) & 1 \\
$\frac{\pi}{2}$-rotation within the lattice & $(-1)^{\frac{N}{4}+n}$ \\
mirror with respect to the $x$-link & 1 \\
translations & 1 \\
\hline \hline
\end{tabular}

To obtain the $d$-wave character from this identity, expand the projected planar state into each $S_{z}$-subspace. Under Eq. (59), it takes a form,

$$
\begin{aligned}
& \left|\Psi_{\text {planar }}\right\rangle=\cdots+\mathcal{P}_{S_{z}=-2}\left|\Psi_{\text {planar }}\right\rangle+\mathcal{P}_{S_{z}=0}\left|\Psi_{\text {planar }}\right\rangle \\
& \quad+\mathcal{P}_{S_{z}=2}\left|\Psi_{\text {planar }}\right\rangle+\mathcal{P}_{S_{z}=4}\left|\Psi_{\text {planar }}\right\rangle+\cdots
\end{aligned}
$$

for $N=4 l(l=1,2, \cdots)$ spins. Among this set of states, the quadrupole operator connects only those two states whose $S_{z}$ differ by 2 ,

$$
\begin{aligned}
& f(\boldsymbol{j}-\boldsymbol{m})=\sum_{n} \\
& \left\langle\Psi_{\text {planar }}\left|\mathcal{P}_{S_{z}=2 n+2} S_{\boldsymbol{j},+} S_{\boldsymbol{m},+} \mathcal{P}_{S_{z}=2 n}\right| \Psi_{\text {planar }}\right\rangle .
\end{aligned}
$$

From Eq. (65), one of these two states is always even under the space $\frac{\pi}{2}$-rotation, whereas the other is odd. This clearly assigns the $d$-wave spatial configuration of the quadrupole moments, i.e. Eq. 63].

The arguments so far also suggest how to construct a trial wavefunction for the so-called Anderson's tower of states (or quasi-degenerate joint states) of the symmetry breaking $d$-wave spin nematic order under the field. For $N=4 l$ spin clusters, $\mathcal{P}_{S_{z}=2 n}\left|\Psi_{\text {planar }}\right\rangle$ mimic the quasidegenerate joint states (QDJS) of the spin nematic ordered phase, whose representation under the point group symmetry operators are listed in Table. I. These representations are actually consistent with those of the BoseEinstein condensate phase of a two-magnon bound state near the saturation field $\underline{\underline{9}}$

The projected $Z_{2}$ planar state in the zero-field case is time-reversal symmetric; the wavefunction derived from Eq. (11) preserves the following symmetry property,

$$
\begin{aligned}
& \operatorname{Pf}\left[\boldsymbol{X}_{\text {planar }}\left(\left\{\sigma_{\boldsymbol{n}}\right\}\right)\right]^{*} \\
& =(-1)^{\frac{N}{2}}\left\{\prod_{\boldsymbol{j}}(-1)^{\sigma_{j}}\right\} \operatorname{Pf}\left[\boldsymbol{X}_{\text {planar }}\left(\left\{-\sigma_{\boldsymbol{n}}\right\}\right)\right] .
\end{aligned}
$$

To see this relation, notice first that the time-reversal operation changes the sign of the triplet Cooper pairing fields in Eq. (11), while does not affect the singlet pairing fields. Such a sign change can be readily compensated by the previous staggered gauge transformation, $f_{j}^{\dagger} \rightarrow$ $f_{j}^{\dagger}(-1)^{j_{x}+j_{y}}$, which imposes the following relation onto the BCS gap functions:

$$
\left[\boldsymbol{t}_{\boldsymbol{k}}\right]^{*}=\boldsymbol{\sigma}_{2}\left[\boldsymbol{t}_{-\boldsymbol{k}+(\pi, \pi)}\right] \boldsymbol{\sigma}_{2} .
$$



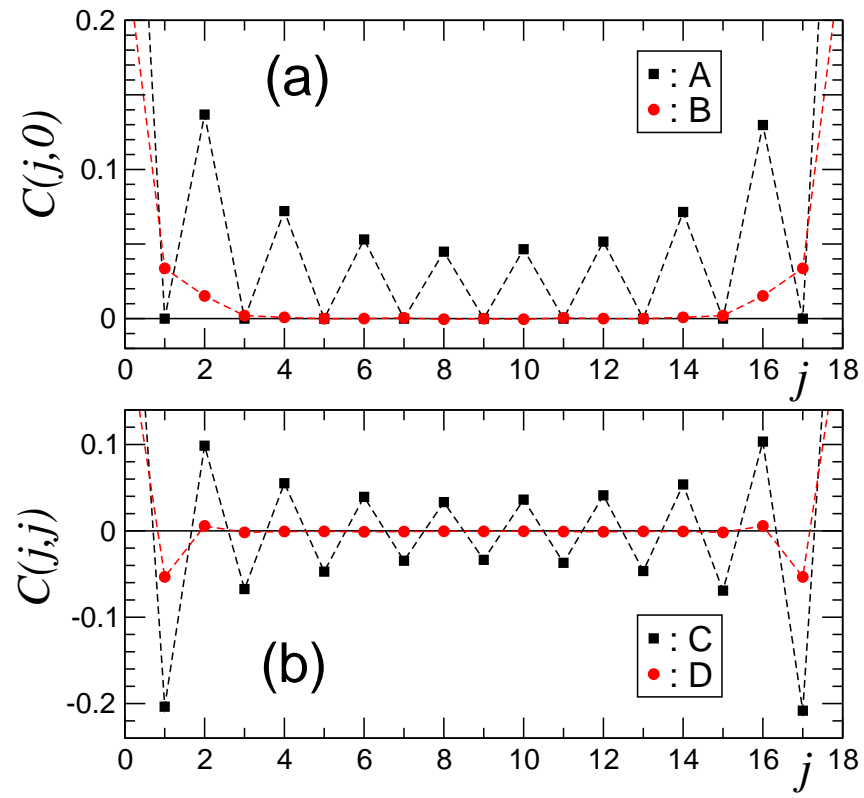

FIG. 6: (Color online) Spin correlation functions of the transverse component $C_{ \pm}(\boldsymbol{j})$ (A and $\mathrm{C}$ ) and the longitudinal one $C_{z z}(\boldsymbol{j})$ (B and $\mathrm{D}$ ) in the projected $Z_{2}$ planar state $\mathcal{P}_{S_{z}=0}\left|\Psi_{\text {planar }}\right\rangle$ along (a) the $x$-direction $(1,0)$ and (b) the diagonal direction $(1,1) . \quad\left[\mathrm{A}: C_{ \pm}(j, 0), \mathrm{B}: C_{z z}(j, 0), \mathrm{C}\right.$ : $C_{ \pm}(j, j)$ and D: $\left.C_{z z}(j, j).\right]$ The projected planar state was obtained in the $J_{1}-J_{2}$ model with $J_{1}=-1$ and $J_{2}=0.45$ in $18 \times 18$ spin cluster, which takes the variational parameters as $(D, \chi, \eta)=(0.40,0.57,0.72)$. The error-bar is smaller than the symbol.

Or equivalently,

$$
\begin{aligned}
& {\left[\boldsymbol{X}_{\text {planar }}\left(\left\{\sigma_{\boldsymbol{n}}\right\}\right)\right]_{j m}^{*}=(-1)^{j_{x}+j_{y}}(-1)^{m_{x}+m_{y}} } \\
& \times(-1)^{\sigma_{j}}(-1)^{\sigma_{m}}\left[\boldsymbol{X}_{\text {planar }}\left(\left\{-\sigma_{\boldsymbol{n}}\right\}\right)\right]_{\boldsymbol{j} \boldsymbol{m}}
\end{aligned}
$$

Noting that $\operatorname{Pf}\left[\boldsymbol{O}^{T} \boldsymbol{A} \boldsymbol{O}\right]=\operatorname{det} \boldsymbol{O} \operatorname{Pf}[\boldsymbol{A}]$, one immediately obtain Eq. (67). This equation especially means that, as far as $\left|\Psi_{\text {planar }}\right\rangle$ is constructed from Eq. (11), both $\mathcal{P}_{S_{z}=0}\left|\Psi_{\text {planar }}\right\rangle$ and $\mathcal{P}_{S=0}\left|\Psi_{\text {planar }}\right\rangle$ are even under the time reversal operation for $N=4 l$ spin systems, which is also consistent with the nature of the spin nematic phase suggested by the previous exact diagonalization studies. $\underline{9}$

\section{STATIC CORRELATION FUNCTIONS}

Based on energy comparison and symmetry arguments, we have argued so far that the projected $Z_{2}$ planar state is likely to be realized in the square lattice $S=1 / 2$ $J_{1}-J_{2}$ frustrated ferromagnetic model in the intermediate coupling range $0.417\left|J_{1}\right| \lesssim J_{2} \lesssim 0.57\left|J_{1}\right|$. To give a direct physical characterization to this intermediate phase, we discuss in this section the static correlation functions calculated with respect to $\mathcal{P}_{S_{z}=0}\left|\Psi_{\text {planar }}\right\rangle$ and $\mathcal{P}_{S=0}\left|\Psi_{\text {planar }}\right\rangle$. From the energetics, it is clear that $\mathcal{P}_{S=0}\left|\Psi_{\text {planar }}\right\rangle$ is closer to the symmetric ground state of finite spin clusters than the other in the intermediate phase. On the other hand, the correlation function of $\mathcal{P}_{S_{z}=0}\left|\Psi_{\text {planar }}\right\rangle$ offers a feature of spin-rotational symmetry broken spin nematic state.

Let us begin with the spin correlation function calculated with respect to $\mathcal{P}_{S_{z}=0}\left|\Psi_{\text {planar }}\right\rangle$. In Fig. 6, we show the characteristic behavior of the transverse component of the spin correlation function (labeled as ' $\mathrm{A}$ ' and ' $\mathrm{C}$ '),

$$
\begin{aligned}
C_{+-}(\boldsymbol{j}-\boldsymbol{m})=\frac{1}{2}\langle & \Psi_{\text {planar }} \mid \mathcal{P}_{S_{z}=0}\left\{\hat{S}_{\boldsymbol{j},+} \hat{S}_{\boldsymbol{m},-}\right. \\
& \left.+\hat{S}_{\boldsymbol{j},-} \hat{S}_{\boldsymbol{m},+}\right\} \mathcal{P}_{S_{z}=0}\left|\Psi_{\text {planar }}\right\rangle,
\end{aligned}
$$

and the longitudinal one (labeled as ' $\mathrm{B}$ ' and ' $\mathrm{D}$ '),

$$
C_{z z}(\boldsymbol{j}-\boldsymbol{m})=\left\langle\Psi_{\text {planar }}\left|\mathcal{P}_{S_{z}=0} \hat{S}_{\boldsymbol{j}, z} \hat{S}_{\boldsymbol{m}, z} \mathcal{P}_{S_{z}=0}\right| \Psi_{\text {planar }}\right\rangle .
$$

Observing them, notice first that the transverse component of the spin in the $A$-sublattice in which $j_{x}+j_{y}$ is even has no correlation at all with those in the $B$-sublattice in which $j_{x}+j_{y}$ is odd. More generally, this feature holds true for any (projected) $Z_{2}$ planar state derived from Eq. (11) or (13), i.e.,

$$
\left\langle\Psi_{\text {planar }}\left|\left\{\hat{S}_{\boldsymbol{j},+} \hat{S}_{\boldsymbol{m},-}+\hat{S}_{\boldsymbol{j},-} \hat{S}_{\boldsymbol{m},+}\right\}\right| \Psi_{\text {planar }}\right\rangle=0
$$

for $\forall \boldsymbol{j} \in A$ and $\forall \boldsymbol{m} \in B$. Equation (68) can be understood from the symmetry argument. Suppose that the spin $\theta$-rotation around the $z$-axis is applied onto all the spins in the $A$-sublattice, while the spin $-\theta$ rotation is in the $B$-sublattice. The mean-field Hamiltonian for the $Z_{2}$ planar state is invariant under this continuous transformation, so that the staggered magnetization $S_{A, z}-S_{B, z}=\frac{1}{2} \sum_{\boldsymbol{j} \in A} \sigma_{\boldsymbol{j}}-\frac{1}{2} \sum_{\boldsymbol{j} \in B} \sigma_{\boldsymbol{j}}$ is a conserved quantity. Applying this staggered spin rotation to the projected $Z_{2}$ planar states, we obtain

$$
\left\langle\left\{\sigma_{j}\right\} \mid \Psi_{\text {planar }}\right\rangle=e^{i \theta\left(S_{A, z}-S_{B, z}\right)}\left\langle\left\{\sigma_{j}\right\} \mid \Psi_{\text {planar }}\right\rangle
$$

for any $\theta$. Equivalently, we have

$$
\left\langle\left\{\sigma_{j}\right\} \mid \Psi_{\text {planar }}\right\rangle=\delta_{S_{A, z}, S_{B, z}} g\left(\left\{\sigma_{j}\right\}\right),
$$

which immediately leads to Eq. (68).

Equation (68) suggests that, as for the transverse component of the spin correlation function, the next-nearestneighbor antiferromagnetic interaction dominates over the competing nearest-neighbor ferromagnetic interaction. In fact, the transverse spin exhibits a strong antiferromagnetic correlation on each of the 'unfrustrated' sublattice (square lattice with $J_{2}$ bonds), where the function decays nearly in a power-law. (see ' $\mathrm{A}$ ' and ' $\mathrm{C}$ ' in Fig. 6). On the one hand, the longitudinal component always has a ferromagnetic correlation between the nearest neighbor spins and an antiferromagnetic correlation between the 2nd neighbor spins. Thus, the spin-frustration among the ferromagnetic bonds and antiferromagnetic ones effectively suppresses the overall amplitude of the longitudinal correlation function (see ' $\mathrm{B}$ ' and ' $\mathrm{D}$ ' in Fig. 6). Indeed, $C_{z z}(\boldsymbol{j})$ decays quite rapidly and falls below $10^{-2}$ 

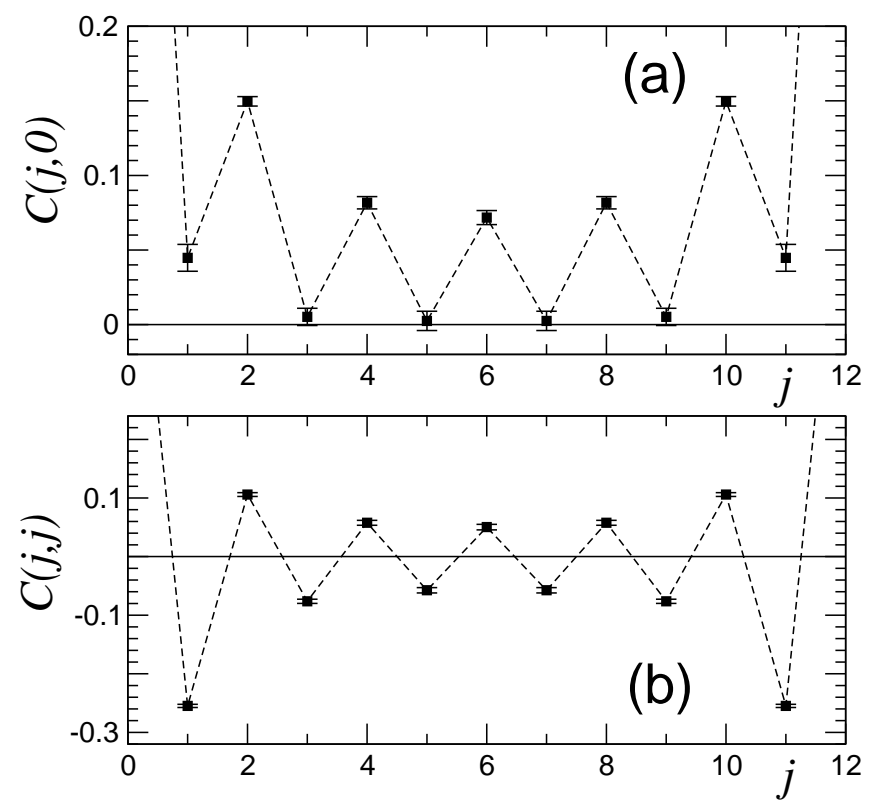

FIG. 7: Spin correlation function $C(\boldsymbol{j})$ in the spin-singlet state $\mathcal{P}_{S=0}\left|\Psi_{\text {planar }}\right\rangle$ along the $x$-direction (a) and along the diagonal direction (b); [(a) $C(j, 0)$ and (b) $C(j, j)]$. The projected planar state was obtained for the $J_{1}-J_{2}$ model with $J_{2}=0.45\left|J_{1}\right|$ in $12 \times 12$ spin cluster, which takes the variational parameters as $(D, \chi, \eta)=(0.45,0.55,0.70)$.

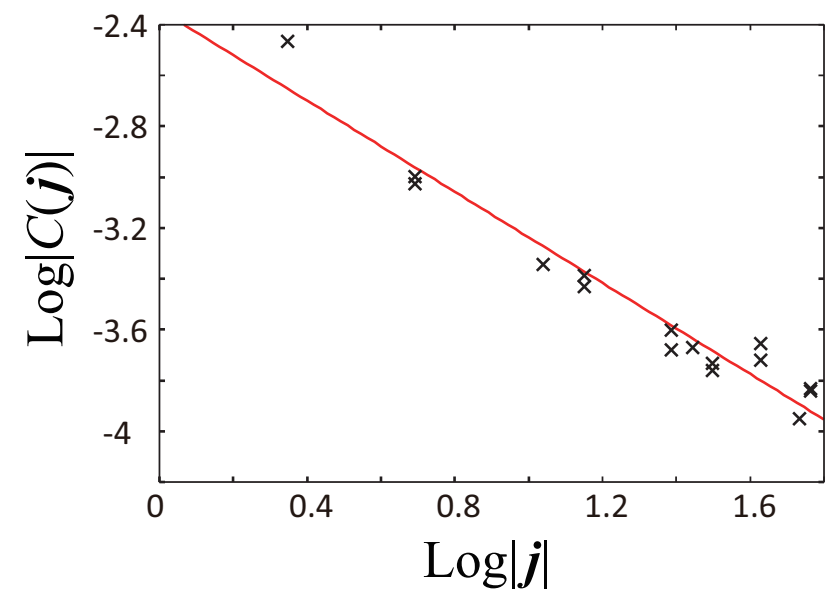

FIG. 8: (Color online) A log-log plot of the correlation function $C(\boldsymbol{j})$ in the spin-singlet state $\mathcal{P}_{S=0}\left|\Psi_{\text {planar }}\right\rangle$ with the same parameter set as used in Fig. 7 We employ only those points with $\max \left(j_{x}, j_{y}\right) \leq 6$ and $j_{x}+j_{y}=$ even. The red solid line indicates the slope of $C(\boldsymbol{j}) \sim(-1)^{j_{x}}|\boldsymbol{j}|^{-0.9}$.

when the spins are spatially separated by more than two sites, $|\boldsymbol{j}|>3$.

When the wavefunction is projected onto the spin singlet space, the static spin correlation function

$$
C(\boldsymbol{j}-\boldsymbol{m})=\left\langle\Psi_{\text {planar }}\left|\mathcal{P}_{S=0} \hat{\boldsymbol{S}}_{\boldsymbol{j}} \cdot \hat{\boldsymbol{S}}_{\boldsymbol{m}} \mathcal{P}_{S=0}\right| \Psi_{\text {planar }}\right\rangle
$$

becomes spin-rotational invariant, which 'interpolate' between $C_{+-}(\boldsymbol{j})$ and $C_{z z}(\boldsymbol{j})$ described above. Namely, as
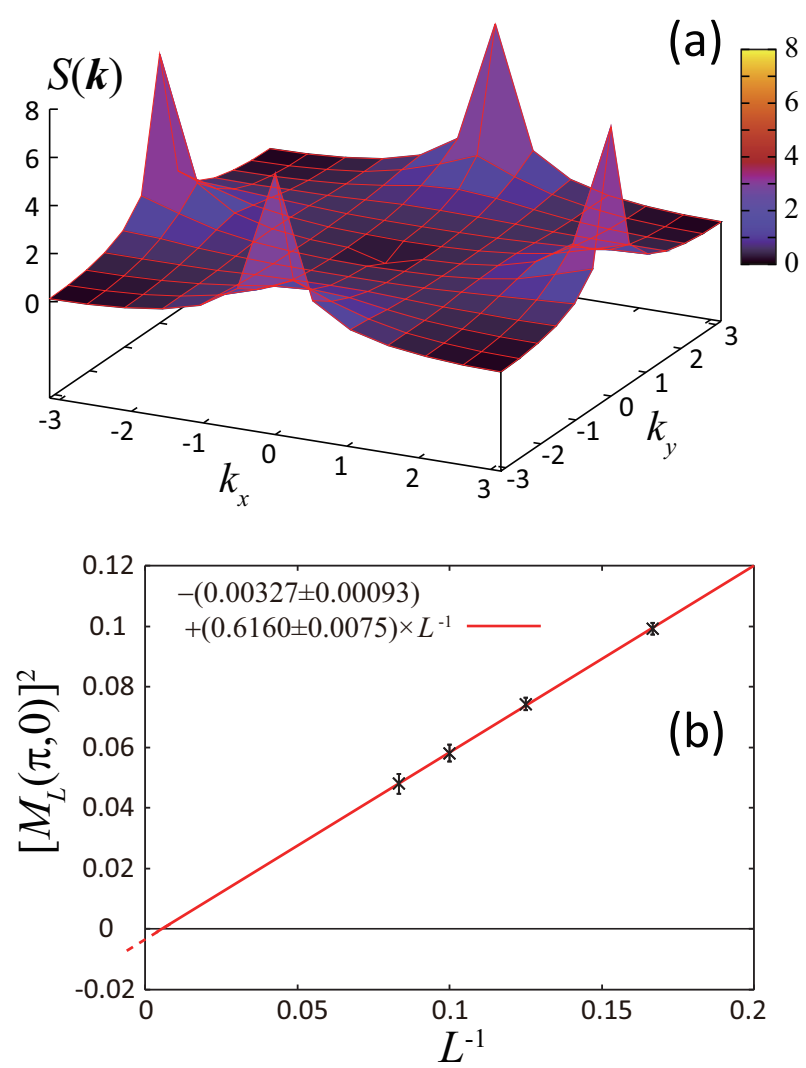

FIG. 9: (Color online) (a) Static spin structure factor $S(\boldsymbol{k})$ calculated from $\mathcal{P}_{S=0}\left|\Psi_{\text {planar }}\right\rangle$ with the same parameter set as used in Fig. 7 . The momentum vector $\boldsymbol{k}$ ranges over the 1st Brillouin zone, $[-\pi, \pi] \times[-\pi, \pi]$. (b) Finite size scaling of $\left[M_{L}(\pi, 0)\right]^{2}=\frac{1}{N+2} S(\pi, 0)$ as a function of the linear dimension of the system size, indicating that $M_{L}(\pi, 0)$ converges to zero in the thermodynamic limit within the statistical error of the Monte Carlo estimation.

shown in Fig. 7. the correlation between the spins in the $A$-sublattice and those in the $B$-sublattice are either extremely short-range or almost quenched, while the correlation within each sublattice exhibits an antiferromagnetic 'quasi-long-ranged' power law decay, which is fitted as $C(\boldsymbol{j}) \sim(-1)^{j_{x}}|\boldsymbol{j}|^{-\eta}$ with $\eta=0.9 \sim 1.0$ for $j_{x}+j_{y}=$ even (see Fig. 8). Correspondingly, the static spin structure factor calculated with respect to $\mathcal{P}_{S=0}\left|\Psi_{\text {planar }}\right\rangle$

$$
S(\boldsymbol{k})=\sum_{\boldsymbol{j}} e^{i \boldsymbol{k} \cdot \boldsymbol{j}} C(\boldsymbol{j})
$$

has characteristic peaks at $(\pi, 0)$ and $(0, \pi)$, while it lose its weight at $(0,0)$ and $(\pi, \pi)$ (see Fig. 9(a)). This behavior resembles the structure factor in the collinear antiferromagnetic phase and seems to be consistent with a recent exact diagonalization study up to 40 sites. ${ }^{20}$ In spite of the prominent antiferromagnetic fluctuation at $(0, \pi)$ and $(\pi, 0)$, however, the standard finite size scaling fitting of the static spin structure factor ${ }^{44,45}$ suggests that the projected $Z_{2}$ planar state does not have any finite 

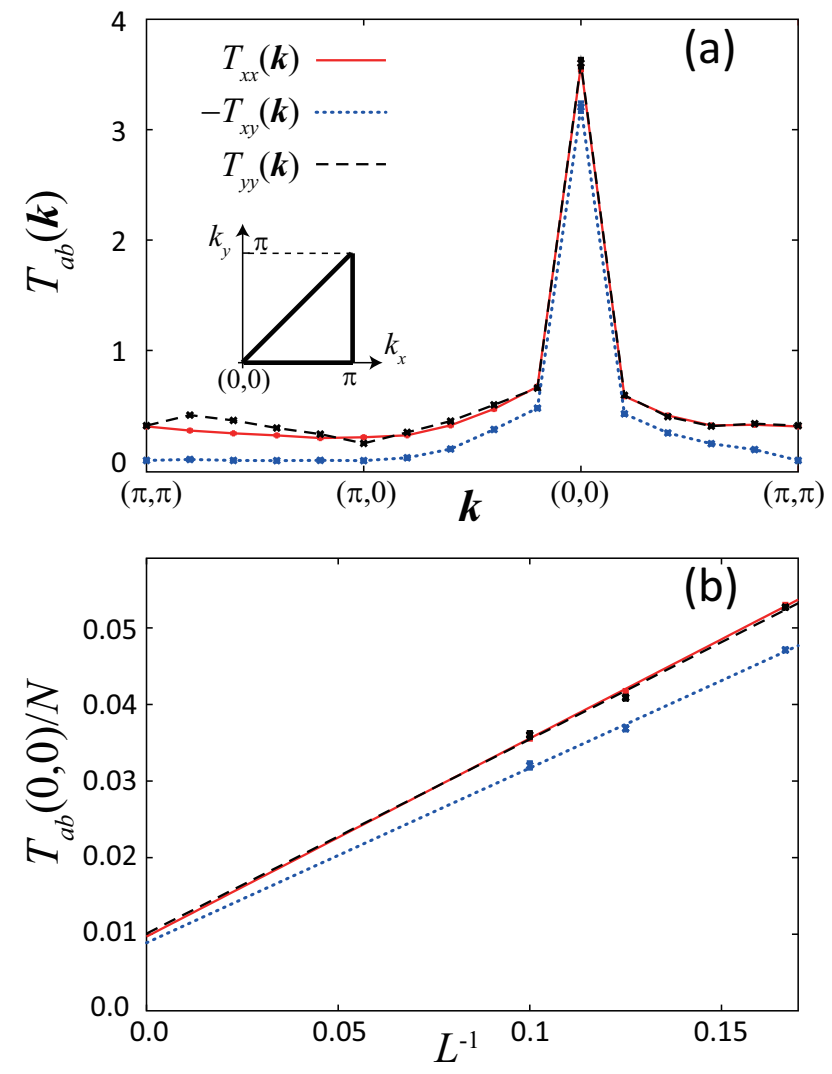

FIG. 10: (Color online) (a) Static structure factor $T_{a b}(\boldsymbol{k})$ of the quadrupole moments calculated in the projected $Z_{2}$ planar state $\mathcal{P}_{S=0}\left|\Psi_{\text {planar }}\right\rangle$, with the same parameter set as used in Fig. 7 for the $J_{1}-J_{2}$ model with $J_{2}=0.45\left|J_{1}\right|$ in $10 \times 10$ spin cluster. The (red) solid line depicts the diagonal component $T_{x x}(\boldsymbol{k})$, the (blue) dotted line the off-diagonal component with minus sign $-T_{x y}(\boldsymbol{k})$, and the (black) dashed line the other diagonal component $T_{y y}(\boldsymbol{k})$. Inset: The momentum $\boldsymbol{k}$ is taken along the high-symmetric momentum points. (b) Finite size scalings of the long-range order of quadrupole moments $\frac{1}{N} T_{a b}(0,0)$ as a function of $1 / L$. The off-diagonal component $T_{x y}(0,0)$ is shown multiplied by minus sign.

sublattice magnetization $M(\pi, 0)$ in the thermodynamic limit [see Fig. 9(b)];

$$
[M(\pi, 0)]^{2}=\lim _{L \rightarrow \infty} \frac{1}{L^{2}+2} \sum_{j} C(\boldsymbol{j}) e^{i \pi j_{x}} \simeq 0 .
$$

The correlation functions of the quadrupole moments are calculated in terms of the projected $Z_{2}$ planar state in the spin-singlet subspace,

$$
\begin{aligned}
D_{a b}(\boldsymbol{j}-\boldsymbol{m})=\sum_{\mu, \nu=1}^{3} & \left\langle\Psi_{\text {planar }}\right| \mathcal{P}_{S=0} K_{\boldsymbol{j}, \boldsymbol{j}+\boldsymbol{e}_{a}}^{\mu \nu} \\
& \times K_{\boldsymbol{m}, \boldsymbol{m}+\boldsymbol{e}_{b}}^{\nu \mu} \mathcal{P}_{S=0}\left|\Psi_{\text {planar }}\right\rangle
\end{aligned}
$$

with $a, b=x, y$. We found that the diagonal components thus calculated, $D_{x x}(\boldsymbol{j})$ and $D_{y y}(\boldsymbol{j})$, are positive-definite for any $\boldsymbol{j}$, while the off-diagonal component always takes a negative value, $D_{x y}(\boldsymbol{j})<0$. In the static structure factor of the quadrupole moments

$$
T_{a b}(\boldsymbol{k})=\sum_{\boldsymbol{j}} e^{i \boldsymbol{k} \cdot \boldsymbol{j}} D_{a b}(\boldsymbol{j}),
$$

both the diagonal components and (minus of) the offdiagonal component exhibit prominent peaks at the $\Gamma$ point [see Fig. 10(a)], indicating the $d$-wave ordering character of the quadrupole moments in the projected $Z_{2}$ planar state. In fact, finite size scalings of their peak values suggest that the state is indeed accompanied by finite quadrupole moments in the thermodynamic limit [see Fig. 10(b)].

\section{SUMMARY AND DISCUSSION}

In this paper, we have investigated the phase diagram and the nature of a quantum spin nematic phase in the spin- $\frac{1}{2}$ quantum frustrated $J_{1}-J_{2}$ model with ferromagnetic $J_{1}$ on the square lattice, describing the ground state wavefunction in terms of a spin-triplet pairing state of the spinon fields. Our theory is based on the previous fermionic mean-field analysis, $\frac{18}{\underline{1}}$ which proposed four types of the mean-field solutions. These solutions include (i) $Z_{2}$ planar state, (ii) $Z_{2}$ polar state, (iii) $S U(2)$ chiral $p$-wave state and (iv) 'flat-band' state, all of which are characterized by different kinds of spin-triplet pairings of the spinon fields introduced on ferromagnetic bonds. Like in usual 'projective description' of symmetric quantum spin liquids,$\frac{1,26,31,32,41}{2}$ we construct projected BCS wavefunctions out of these triplet pairing states. Performing variational Monte Carlo simulations based on these projected wavefunctions, we obtain the phase diagram Fig. 1 and static correlation functions in the spin nematic wavefunction.

We first argue how these mean-field pairing states are deformed, when external Zeeman field is applied. A direct minimization of the mean-field energy dictates that all the $d$-vectors in these pairing states are restricted within a plane perpendicular to the applied field. This arrangement makes these pairing states invariant under the spin $\pi$-rotation around the magnetic field. Owing to this $\pi$-rotational symmetry, the corresponding projected BCS wavefunctions acquire the 'spin-nematic' character; ordering of (the transverse component of) the quadrupole moments without any spontaneous ordering of (the transverse) spin moments nor any magnetic crystallization. We also show that the 'flat-band' state, which achieves the lowest mean-field energy in the strong ferromagnetic regime $\left|J_{1}\right| \gg J_{2}$, actually ends up in the trivial fully polarized state, when projected onto the spin Hilbert space.

To examine a possible realization of quantum spin nematic states in the present spin model, we study the energetics of the projected (i) $Z_{2}$ planar state, (ii) $Z_{2}$ polar state, and (iii) $S U(2)$ chiral $p$-wave state. We focus especially on the intermediate coupling regime, where the ferromagnetic exchange $J_{1}$ is about twice as large as the 
antiferromagnetic exchange $J_{2}, J_{2} \simeq 0.5\left|J_{1}\right|$. Based on the variational Monte Carlo analysis, we argued that, in a finite range of this intermediate coupling regime, the projected $Z_{2}$ planar state achieves the best optimized energy, compared with the energies of other competing phases, such as the ferromagnetic phase and collinear antiferromagnetic phase. (See Fig. 1) We also prove that this projected $Z_{2}$ planar state is accompanied by the ' $d$ wave' spatial configuration of the quadrupole moments. This feature of the wavefunction including the irreducible representations under the symmetry group turns out to show a perfect agreement with the nature of the quantum spin nematic phase suggested by Shannon et al. ${ }^{9}$ from the exact diagonalization study.

Motivated by this coincidence, we further calculate the spin-spin correlation function of the projected $Z_{2}$ planar state, so as to obtain the static spin structure factor in the spin nematic phase. The structure factor thus calculated exhibits two prominent peaks at the wavevectors $\boldsymbol{k}=(\pi, 0)$ and $(0, \pi)$, which signifies the presence of strong collinear antiferromagnetic fluctuation. The finite size scaling of the peak height concludes that the state does not possess any sublattice magnetization in the thermodynamic limit, which is consistent with the spin-nematic feature of the $Z_{2}$ planar state. This antiferromagnetic fluctuation is reminiscent of the neighboring collinear antiferromagnetic phase, whereas ferromagnetic fluctuation, which is also expected to appear from the other neighboring ferromagnetic phase, is completely suppressed.

These observations indicate that the static spin-spin correlation function by itself hardly distinguishes the current quantum spin nematic phase from the neighboring collinear antiferromagnetic phase. Indeed, the recent exact diagonalization study by Richter et al. 20 reported that the present $J_{1}-J_{2}$ model exhibits only a strong collinear antiferromagnetic correlation in the intermediate coupling regime, $J_{2} \simeq 0.5\left|J_{1}\right|$. We expect that the dynamical magnetic properties in combination with these static physical properties could distinguish the present spin nematic phase from the collinear antiferromagnetic phase. That is, unlike in the collinear antiferromagnetic phase, all the gapless Goldstone modes in the spin-nematic phase are expected to lose their spectral weight in the dynamical spin structure factor in the lowenergy limit. In fact, a recent calculation based on the random phase approximation shows that this is indeed the case in the $Z_{2}$ planar state. ${ }^{43}$ This feature could be sharply contrasted to the dynamical magnetic property in the collinear antiferromagnetic phase, where the gapless mode at the $(0, \pi)$-point or $(\pi, 0)$-point have a finite spectral weight even in the low-energy limit.

\section{Acknowledgments}

We acknowledge Masatoshi Imada, Yukitoshi Motome, Takashi Koretsune, Philippe Sindzingre, and Leon Balents for helpful discussions. RS was partially supported by the Institute of Physical and Chemical Research (RIKEN). This work was supported by Grantsin-Aid for Scientific Research from MEXT, Japan (No. 22014016 and No. 23540397).
${ }^{1}$ X. G. Wen, Quantum Field Theory of Many-Body Systems, (Oxford Unviersity Press, Oxford, 2004).

2 P. Fazekas and P. W. Anderson, Philosophical Magazine 30, 423-440 (1974); P. W. Anderson, Science 235, 1196 (1987).

3 L. Balents, Nature 464, 199 (2010); P. A. Lee, Science 321, 1306, (2008).

4 G. Misguich and C. Lhuillier, in Frsutrated Spin Systems, edited by H. T. Diep (World Scientific, Singapore, 2004).

5 K. Ishida, M. Morishita, K. Yawata, and Hiroshi Fukuyama, Phys. Rev. Lett. 79, 3451 (1997).

6 R. Masutomi, Y. Karaki, and H. Ishimoto, Phys. Rev. Lett. 92, 025301 (2004).

7 M. Roger, J. M. Delrieu, and J. H. Hetherington, Phys. Rev. lett. 45, 137 (1980); Rev. Mod. Phys. 55, 1 (1983).

8 A. F. Andreev and I. A. Grishchuk, Sov. Phys. JETP 60, 267 (1984).

9 N. Shannon, T. Momoi, and P. Sindzingre, Phys. Rev. Lett. 96, 027213 (2006); P. Sindzingre, N. Shannon, and T. Momoi, J. Magn. Magn. Mater. 310, 1340 (2007).

10 P. Sindingre, L. Seabra, N. Shannon, and T. Momoi, J. Phys. Conf. Ser. 145, 012048 (2009).

11 P. Sindzingre, N. Shannon, and T. Momoi, J. Phys. Conf. Ser. 200, 022058 (2010).

12 T. Momoi and N. Shannon, Prog. Theor. Phys. Suppl. 159,
$72(2005)$

13 T. Momoi, P. Sindzingre, and N. Shannon, Phys. Rev. Lett. 97, 257204 (2006).

14 D. V. Dmitriev, V. Ya. Krivnov, and A. A. Ovchinnikov, Phys. Rev. B 55, 3620 (1997).

15 N. Shannon, B. Schmidt, K. Penc, and P. Thalmeier, Eur. Phys. J. B 38, 599 (2004).

16 B. Schmidt, N. Shannon, and P. Thalmeier, J. Phys.: Condens. Matter 19, 145211 (2007); J. Magn. Magn. Mater. 310, 1231 (2007).

17 J. R. Viana and J. R. de Souza, Phys. Rev. B 75, 052403 (2007).

18 R. Shindou and T. Momoi, Phys. Rev. B 80, 064410 (2009).

19 M. Härtel, J. Richter, D. Ihle, and S.-L. Drechsler Phys. Rev. B 81, 174421 (2010).

20 J. Richter, R. Darradi, J. Schulenburg, D. J. J. Farnell, and H. Rosner, Phys. Rev. B 81, 177429 (2010).

21 P. Chandra, P. Coleman, and A. I. Larkin, Phys. Rev. Lett. 64, 88 (1990).

22 A. V. Chubukov, Phys. Rev. B 44, 4693 (1991).

23 P. Chandra and P. Coleman, Phys. Rev. Lett. 66, 100 (1991); P. Chandra, P. Coleman, and A. I. Larkin, J. Phys. Condens. Matter 2, 7933 (1990).

24 E. Fradkin, Field Theories of Condensed Matter Systems 
(Addison-Wesley, London, 1991).

25 Assa Auerbach, Interacting electrons and Quantum Magnetism (Springer-Verlag, New York, 1994).

${ }^{26}$ G. Baskaran, Z. Zou, P. W. Anderson, Solid State Communication 63, 973 (1987); G. Baskaran and P. W. Anderson Phys. Rev. B 37, 580 (1988); E. Dagotto, E. Fradkin, and A. Moreo, Phys. Rev. B 38, 2926 (1988).

27 Quantum spin states with spin-triplet pairing of fermions are explored also in $S \geq 1$ quantum spin systems; ZhengXin Liu, Yi Zhou, and Tai-Kai Ng, Phys. Rev. B 82, 144422 (2010).

${ }^{28}$ In Ref. 18, we called the planar state as the (twodimensional analogue of) Balian-Werthamer state and the polar state as the collinear state. In this paper, we adopt 'planar/polar' instead, since they seem to be more familiar in the standard literature such as Refs. 29 and 30.

29 D. Vollhardt and P. Wolfle, The Superfluids Phase of Helium 3, (Taylor \& Francis, 1990).

30 G. E. Volovik, The universe in a Helium droplet, (Oxford University Press, 2003).

31 J. B. Marston and I. Affleck, Phys. Rev. B 39, 11538 (1989); I. Affleck and J. B. Marston, Phys. Rev. B 37, 3774 (1988).

32 C. Gros, Annals of Physics 189, 53 (1989); B. Edegger, V. N. Muthukumar, and C. Gros, Advances in Physics, 56, 927 (2007).

33 S. Sorella, Phys. Rev. B 64, 024512 (2001); M. Casula and S. Sorella, J. Chem. Phys. 119, 6500 (2003); M. Casula, C. Attaccalite, and S. Sorella, J. Chem. Phys. 121, 7110 (2004); S. Yunoki and S. Sorella, Phys. Rev. B 74, 014408 (2006).

34 Claudia Filippi and Stephen Fahy, J. Chem. Phys. 112,
3523 (2000); C. J. Umrigar and Claudia Filippi, Phys. Rev. Lett. 94, 150201 (2005).

35 J. P. Bouchaud, A. Georges, and C. Lhuillier, J. Phys. (Paris) 49, 553 (1988)

36 M. Bajdich, L. Mitas, L. K. Wagner, and K. E. Schmidt, Phys. Rev. B 77, 115112 (2008).

37 See for example Fig. 3 in Ref. 9 .

38 D. Tahara and M. Imada, J. Phys. Soc. Jpn. 77, 114701 (2008).

39 P. Ring and P. Schuck, The Nuclear Many-Body Problem (Springer, Heidelberg, 1980); T. Mizusaki and M. Imada, Phys. Rev. B 69, 125110 (2004); F. F. Assaad, P. Werner, P. Corboz, E. Gull, and M. Troyer, Phys. Rev. B 72, 224518 (2005); T. Aimi and M. Imada, J. Phys. Soc. Jpn. 76, 084709 (2007).

40 M. Abramowitz and I. A. Stegun, Handbook of Mathematical Functions, Applied Mathematics Series, Vol. 55, 916 (Washington: National Bureau of Standards; reprinted 1968 by Dover Publications, New York).

41 C. Gros, Phys. Rev. B 38, 931 (1988); F. C. Zhang, C. Gros, T. M. Rice, and H. Shiba, Supercond. Sci. Technol. 1, 36 (1988).

42 S. Liang, B. Doucot, and P. W. Anderson, Phys. Rev. Lett, 61, 365 (1988).

43 R. Shindou, S. Yunoki, and T. Momoi, arXiv:XXXX

44 D. Huse, Phys. Rev. B 37, 2380 (R) (1988).

45 H. J. Schulz and T. A. L. Ziman, Europhysics Letter 18, 355 (1992); H. J. Schulz, T. A. L. Ziman, and D. Poilblanc, J. Phys. I 6, 675 (1996); J. Richter and J. Schulenburg, Eur. Phys. J. B 73, 117 (2010). 